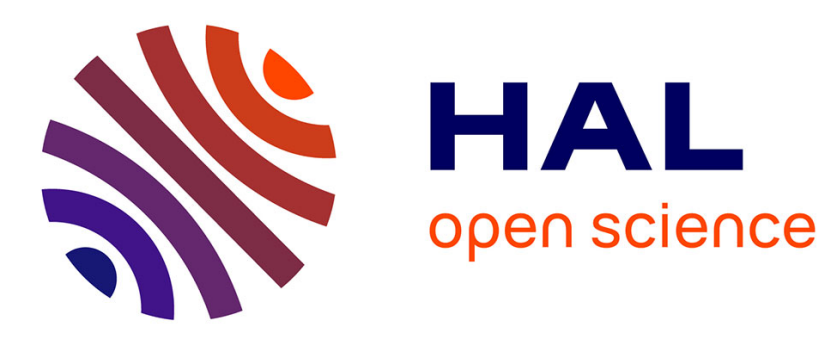

\title{
Electrophysiologic Techniques
}

\author{
Marine Vernet, Shahid Bashir, Syed Faaiz Enam, Hatice Kumru, Alvaro \\ Pascual-Leone
}

\section{To cite this version:}

Marine Vernet, Shahid Bashir, Syed Faaiz Enam, Hatice Kumru, Alvaro Pascual-Leone. Electrophysiologic Techniques. Nathan D. Zasler; Douglas I. Katz; Ross D. Zafonte. Brain injury medicine: principles and practice, 2nd Edition, demosMEDICAL, an inprint of Springer Publishing, pp.230-246, 2012. hal-02947892

\section{HAL Id: hal-02947892 \\ https://hal.science/hal-02947892}

Submitted on 17 Nov 2021

HAL is a multi-disciplinary open access archive for the deposit and dissemination of scientific research documents, whether they are published or not. The documents may come from teaching and research institutions in France or abroad, or from public or private research centers.
L'archive ouverte pluridisciplinaire HAL, est destinée au dépôt et à la diffusion de documents scientifiques de niveau recherche, publiés ou non, émanant des établissements d'enseignement et de recherche français ou étrangers, des laboratoires publics ou privés. 


\section{Traumatic Brain Injury: Electrophysiological Techniques}

\section{Marine VERNET ${ }^{1}$, Shahid BASHIR ${ }^{1,2}$, Syed Faaiz ENAM ${ }^{1,3}$, Hatice KUMRU $^{4}$ and Alvaro PASCUAL-LEONE ${ }^{1,4 *}$}

${ }^{1}$ Berenson-Allen Center for Noninvasive Brain Stimulation, Beth Israel Deaconess Medical Center, Harvard Medical School, 330 Brookline Avenue, KS-158, Boston MA 02215, USA

2 Department of Physiology, Faculty of Medicine, KSU-Autism Research and Treatment Center, King Saud University, P 0 Box 2925, Riyadh 11461, Saudi Arabia

3 Medical College, Aga Khan University Hospital, Stadium Road, P.0 Box 3500, Karachi 74800, Pakistan

${ }^{4}$ Institut Universitari de Neurorehabilitació Guttmann, Universidad Autónoma de Barcelona, Barcelona, Spain.

* Corresponding author: Alvaro Pascual-Leone, M.D., Ph.D. Berenson-Allen Center for Noninvasive Brain Stimulation, Beth Israel Deaconess Medical Center, 330 Brookline Ave, KS-158, Boston MA 02215 (USA). apleone@bidmc.harvard.edu.

Citation: Vernet M, Bashir S, Enam SF, Kumru H, Pascual-Leone A. (2012). Electrophysiologic Techniques. In Zasler N, Zafonte R, Katz D (Eds.), Brain injury medicine: principles and practice (second edition) (Second ed.): Demos Medical. 


\section{INTRODUCTION}

Assessing the extent and functional impact of a traumatic brain injury (TBI), obtaining reliable prognostic indicators, gauging the best therapeutic interventions, and following the course of disease with reliable and objective markers is challenging. Electrophysiological techniques are relatively inexpensive, broadly deployable, repeatable, and safe methods that hold the promise of addressing some of these major clinical needs. Electrophysiological techniques can not only provide continuous and objective monitoring but can also pick up specific functional deficits and pathologies, provide a quantitative scale of severity, and be of great help in guiding rehabilitation and treatment interventions.

Electrophysiological techniques can be used to characterize the brain and central nervous system, as well as various aspects of the peripheral and autonomic nervous system. Evaluation of the peripheral and autonomic systems can be extremely important in patients after TBI because they may reflect consequences of brain injury and offer important prognostic insights. However, the focus of this chapter will be on the role of electrophysiological techniques to assess brain function with the use of electroencephalography (EEG), evoked potentials (EPs), and transcranial magnetic stimulation (TMS) in aiding the diagnosis, prognosis, and therapy of TBI.

\section{ELECTROENCEPHALOGRAPHY}

EEG measures electrical activity of the cerebral cortex through surface electrodes placed on the scalp adhering to standardized placement methods (e.g., the 10-20 International System of Electrode Placement; Figure $1 \mathrm{~A}$ and $1 \mathrm{~B})$. Typical wave frequencies detected include delta (up to $4 \mathrm{~Hz}$ ), theta $(4-8 \mathrm{~Hz}$ ), alpha (8-13 Hz), beta $(13-30 \mathrm{~Hz}$ ) and gamma (above $30 \mathrm{~Hz}$ ). Within each frequency band, different rhythms have been identified and ascribed to different brain/cognitive states (1). The alpha rhythm is a common starting point in the conventional analysis of a clinical EEG, is the dominant rhythm over posterior brain regions, and is attenuated with eye opening. Generally, alpha activity is thought to be related to inhibitory cortical tone and linked to thalamocortical patterns of activation. Mu rhythms are centrally located rhythms in the alpha frequency band that

are attenuated with contralateral movement of an extremity. Beta rhythms are normally activated with mental, lingual, or cognitive efforts, mostly over the frontal areas. Furthermore, many pharmacologic agents increase power in beta band activity, notably benzodiazepines, for example. Theta rhythms can be recorded intermittently over the frontocentral head regions during awake resting or while performing moderately difficult mental tasks; these are enhanced by drowsiness. Delta rhythms are considered a normal finding in the awake state in the very young and in the elderly. They are also considered normal across all ages during slowwave sleep (1). Finally, gamma rhythms are associated with higher cognitive functions involving perception, attention, learning, and memory. These may also serve to assess the temporal dynamics of cortical networks and their interactions 
(2) (Table 17-1).

Abnormalities detected in EEG recordings can indicate primary cortical pathology or be the result of deeper structures modulating cortical regions erratically. In patients with TBI, EEG is one of the electrophysiological techniques often used to assess severity of brain injury and predict prognosis and outcomes (3 - 4). EEG analysis can be divided into conventional and quantitative methods.

\section{Conventional EEG}

Conventional EEG is the standard method for recording cortical electrical waveforms as mentioned earlier (Figure 1C). Although conventional EEG might have some value when assessing injury severity and depth of coma in patients with TBI (5-6), it remains a qualitative tool. Therefore, it does not provide great resolution and cannot quantify wave spectrum frequencies. This makes it impractical for longterm monitoring of patients with TBI and predicting a prognosis (6). Nevertheless, it is often used in neurocritical care for assessment and monitoring of patients with moderate-to-severe TBI (7). Conventional EEG can certainly help in the detection of epileptic activity, a common consequence of more severe TBI. However, use of conventional EEG in early evaluation of patients with mild TBI is rather limited (3).

\section{Mild TBI}

There are no clear EEG features unique to TBI of mild severity (8), and conventional EEG is not reliable in differentiating between mild and moderate TBI either $(4,9)$. There are studies that note an absence of any early EEG abnormalities, (10) even when a structural abnormality is present on magnetic resonance imaging (MRI) (11) or the patient clinically exhibits symptoms of TBI (12-14). However, not all studies report normal conventional EEG following mild TBI or concussion. One study, conducted in 1944 (15), involved the EEG recording of patients with industrial injuries acquired in a shipyard. Most patients, in whom EEG was measured within 15 minutes post-injury, showed little or no apparent alteration in the recording. However, certain patients who experienced the least delay between trauma and EEG recording showed diffuse slowing of EEG activity. This generally resolved within 15 minutes but for some lasted up to an hour. Within the first several hours after mild trauma, attenuated posterior alpha waves (decreased alpha frequency) as well as generalized or focal slow wave activity with a preponderance of theta waves are sometimes observed $(3,8,16-18)$. The presence of these signs may be dependent on the length of loss of consciousness (19). Further, when associated with other signs of complicated injury, these abnormalities predict a poorer prognosis (20). However, the changes are often subtle and sometimes within the range of normal findings in the general population. Even if a longer lasting abnormality is present, it often resolves completely within months after a mild TBI. Correspondence between clinical and EEG findings is relatively poor (8), and any abnormalities discovered tend to resolve during the first several months post-injury (21). In the late period post-injury, approximately $10 \%$ of the individuals tend to show mild EEG 
abnormalities (10). However, the etiology of these is not always clear, and they may not be indicative of brain damage. For example, a low-voltage alpha EEG pattern, months to years after a mild TBI or concussion, is indicative more of anxiety than brain injury (16).

\section{Severe TBI}

The use of EEG in severe TBI is higher than in mild TBI. EEG recordings after severe brain injury correlate well with the depth of post-traumatic coma (22 - 25). During initial stages of a TBI-induced coma, EEG variables such as the amplitude, frequency, and shape of wave potentials are not stable (6). Initial recordings taken within 24 hours post-injury are of less prognostic significance, however, than those from the 24 -to 48 -hour period $(23,26)$. This could be caused by an interplay between both irreversible brain lesions and reversible functional disturbances. The degree of unconsciousness in patients can rapidly change, and thus continuous monitoring has been used for detecting possible signs of clinical deterioration during the first few weeks post-injury (27). Findings, during a post-traumatic coma, range from increased slow activity to amplitude suppression (28). Features typical of sleep, various sharply contoured discharges, epileptic spikes, periodic lateralized epileptiform discharges (PLEDs), and triphasic waves can also be found. However, reactivity and the typical sleep features mentioned earlier are more common among patients who show a good recovery (29).

In the late post-injury period of severe brain injuries, EEGs may show a wide variety of dysrhythmias, focal or generalized suppression, focal slowing, frontal alpha waves, and epileptiform discharges (30-31).

In summary, the use of conventional EEG in mild TBI is limited. Although there are abnormalities sometimes discovered in the EEG of patients with mild TBI, sensitivity is low, and the clinical and functional significance are uncertain. Further, any detected abnormalities may be similar to those present in the general population. Even in the late post-injury period, there is a lot of skepticism toward the significance of epileptiform EEG findings. In severe cases of TBI,

however, the EEG can be more helpful and may even lend a hand in determining a prognosis for the patient.

\section{Post-Traumatic Epilepsy}

Post-traumatic epilepsy (PTE) will be covered in depth in Chapter 39 (Posttraumatic Seizures and Epilepsy) of this book. PTE is a recurrent seizure disorder that results from TBI. PTE is estimated to constitute more than $20 \%$ of cases of symptomatic epilepsy and about 5\% of all cases of epilepsy. PTE must be differentiated from post-traumatic seizures (PTS), which refers to isolated seizures that occur as a sequel to brain injury either within 24 hours (immediate PTS), within 1 week (early PTS), or more than 1 week after injury (late PTS). About $20 \%$ of 
people who have a single late PTS never have any further seizures and should not be labeled as having PTE.

How to predict who will develop epilepsy after TBI and who will not is challenging. The onset of PTE can occur within a short time of the TBI but also months or even years later, and compared with the general population, people with TBI remain at a higher risk for epilepsy even decades after the injury. Serial EEGs may, thus, be helpful in following a patient after TBI and assessing the risk of PTE. However, this practice is not free of challenges.

The severity and type of injury certainly contribute to the risk of developing PTE, for example, penetrating injuries and those causing intracerebral hemorrhages confer a higher risk. On the other hand, development of PTE is a relatively uncommon consequence of mild TBI (32). Nonetheless, a study showed that epileptiform abnormalities assessed with magnetoencephalography (MEG) were present in 10\% of the cases long after an episode of mild TBI (33). However, this statistic may not differ much from the prevalence of these abnormalities in the general population. Indeed, an earlier study (14) reported that 6 months after a mild TBI, the number of patients with epileptiform EEG abnormalities were equal to those who had sustained only a whiplash injury. However, the authors did notice that posttraumatic epileptiform abnormalities increased as time passed while other EEG abnormalities did not. It should be noted that epileptiform activity could also be observed in healthy subjects with no history of seizures. In addition to various epileptiform variant patterns that are nonepileptic in nature $(1,34,35)$, spontaneous interictal epileptiform discharges (IED) can be recorded in healthy volunteers (35). Overall, spontaneous IED rates appear to be higher in patients who are nonepileptic with TBI than in healthy adults $(2 \%-12 \%$ vs $0 \%-6.6 \%)$, and rates for a seizure after IED detection are also higher in patients than in healthy adults (up to $14 \%$ in patients vs $2 \%$ in healthy adults).

It is, thus, difficult to predict the occurrence of PTE when based only on the recording of spontaneous IEDs, particularly after mild TBI. It is usually considered that sleep deprivation is an enhancer of epileptic discharges and seizure frequency. However, this could also be because sleep deprivation often occurs in association with physical or emotional stress and substance abuse. When controlling for these factors, sleep deprivation facilitates IEDs but does not seem to affect seizure frequency (36). However, EEGs after sleep deprivation might be a useful indicator of brain damage after TBI, and follow-up imaging studies (computer tomography [CT] or MRI) seem warranted and frequently reveal abnormalities (37).

In case of greater clinical suspicion, admission to an epilepsy monitoring unit (EMU) for prolonged video-EEG monitoring is the best way to confirm and clarify a diagnosis of epilepsy. In patients with suspected diagnosis of PTE, video-EEG monitoring can provide further diagnostic clarification and certainty in about $80 \%$ of the cases. Importantly, about 30\% will be diagnosed as having psychogenic nonepileptic seizures (38). Finally, deep brain recordings might be necessary to 
precisely localize the epileptic focus before surgery in case of intractable epilepsy.

In summary, the development of PTE is rare after mild TBI, although higher following other more severe TBIs (particularly penetrating wounds and those with intracerebral bleeds). Symptoms can develop long time after the TBI and while EEGs can be helpful in serially assessing the relative risk; presence of epileptiform activity in the EEG does not necessarily predict the occurrence of future seizures. However, it might indicate the existence of more significant brain damage.

\section{Quantitative EEG}

With signal processing technology, EEG data can be quantified and objectively analyzed (Figure 1D). Computer-assisted analysis of EEG data, that is, quantitative EEG (QEEG), offers definite advantages over a trained electroencephalographer's eye in identifying the electrophysiological features of TBI (3-4). Although some studies raise questions about the overall validity and accuracy of QEEG findings (8), many discuss the reliability of use in assessing various neurological disorders (9), specifically in diagnosing and classifying the severity of TBI $(4,39)$. Studies have determined multiple QEEG variables known as discriminant functions. Because of their low cost, speed, and objectivity, these can help in predicting functional characteristics and pathologies (40).

Thatcher et al. $(4,41)$ showed that QEEG was very successful in distinguishing and discriminating mild TBI from controls and also from patients with more severe TBI. They were able to achieve a discriminant classification accuracy of $94.8 \%$ in 1989 amongst a population of 608 cases of mild TBI and 108 age-matched controls. In 2001, the sensitivity in discriminating between mild and severe TBI was $95.45 \%$, and specificity was $97.44 \%$. Thatcher et al. went on to propose "big bump theory" stating that pathological residues and/or compensation could be detectable by QEEG even years later after the original trauma. This is analogous to the big bang theory where cosmic radiation is still detected billions of years after the explosion. The study also discovered that the greatest contribution to discriminant function was actually multivariable and consisted of coherence, phase, and amplitude differences. Consensus says that QEEG of TBI cases show an immediate decrease in the mean frequency of alpha waves and a rise in slow focal or diffuse theta activity (8-9). These often later resolve within weeks and months coinciding with clinical improvement (8).

Methods of analysis of continuous EEG recording have been developed to evaluate changes in connectivity between different brain areas after TBI. EEG coherence, that is, correlation between the spectral content of 2 electrodes over time, is believed to reflect the strength of functional interactions between cortical neuraly networks; EEG phase, that is, the time lag between 2 similar activations at different locations, is believed to be linked to the speed of the connection between the 2 areas. TBI has been characterized by a decreased coherence and increased asymmetry (9). However, these coherence changes can be considered nonspecific findings and can 
certainly be found in pathologies other than TBI (8). Kumar et al. (42) showed that patients with mild TBI depicted normal connectivity at rest from 1 to 6 months after their concussion. These patients, however, had impaired verbal and visuospatial working memory tasks. This impairment was associated with decreased frontoparietal, frontotemporal, temporoparietal, and interhemispheric connectivity during working memory performance. Similarly, during an auditory memory activation condition, abnormal frontal connectivity measures within the low and high beta bands (coherence and phase), as well as a shift toward right temporal functioning, have been associated with auditory memory deficits in patients with TBI (43). Thus, abnormalities of functional connectivity, explored during tasks execution, might be more prominent and more sensitive than abnormalities explored during the resting state.

However, impairment in functional connectivity at rest can be revealed with more sophisticated methods. Cao and Sloubounov (44) described a method in which an independent component analysis (ICA) was run to transform multichannel EEG recordings into independent processes. A source reconstruction algorithm followed this transformation. A graph theory analysis was then performed to assess the connectivity between regions of interest (ROIs). This method was applied to athletes, selected for their high risk of concussion, up to 6 months before and 7 days after a sport-related mild TBI. TBI resulted in a decrease in the long-distance connectivity (between frontal areas and other areas of the brain) and significant increase in the short-distance connectivity (within occipital and parietal areas) at rest, which could not be observed when traditional coherence analysis was implemented.

In summary, these studies reveal how the information contained in the EEG signal is rich and can be mathematically processed to quantify abnormalities after severe TBI and also to reveal subtle abnormalities following mild TBI. The most consistent findings, as summarized by Thatcher (45) are (a) reduced power in the higher frequency bands (alpha and above), related to cortical gray matter injury; $(b)$ increased slow waves in the delta frequency band in severe TBI, which are related to cerebral white matter injury; and $(c)$ changes in EEG coherence and phase delays, related to gray and white matter injury, especially in frontal and temporal lobes. Novel technology that enables wearable, modular, and wireless recording of EEG (Figure 2) on the one hand, and the advent of more powerful, faster analysis algorithms on the other hand, promise to further increase the use of quantitative EEG in TBI.

\section{On the Use of EEG Versus Quantitative EEG: Clinical and Forensic Considerations}

In a report of the American Academy of Neurology and the American Clinical Neurophysiology Society on the assessment of digital EEG, QEEG, and EEG brain mapping published in 1997 (still holding in 2006), it was stated that QEEG remains investigational for clinical use in post-concussion syndrome resulting from mild or 
moderate head injury (46). This statement was criticized in later publications (e.g., 9,47 - 48). The superiority of visual examination over QEEG defended by the American Academy of Neurology is questioned in regard (among others) to the demonstrated subjectivity of visual examination and the large amount of publications based on QEEG. Discriminate analysis with QEEG is also challenging given the fact that frequently, the issue is not simply a differentiation between "TBI" vs "no TBI," that is, patients may have prior mental health issues, post-injury posttraumatic stress disorder (PTSD), depression, anxiety, drug abuse, alcohol, medications, and so forth. In addition, there is ongoing controversy regarding the various normative databases used for QEEG analysis. Thus, QEEG has not become fully established in the clinical realm, yet it can play a role in the medicolegal arena, where it can find some acceptance in courts and for third-party reimbursement (45). Part XIX (Medicolegal and Ethical Issues) of this book will cover in depth these forensic considerations.

\section{EEG Biofeedback}

EEG biofeedback will be covered in depth in chapter 76 (Complementary and Alternative Medicine) of this book. Here, we shall just provide some basic descriptions of the principles. EEG biofeedback offers the opportunity for EEG to go from diagnostic and prognostic applications to therapeutics. Biofeedback techniques have been used to promote improvement of cognitive functions. Biofeedback consists of measuring certain physiologic parameters from a patient and then converting them into a sensorial feedback that is provided to the patient. The feedback is positive (reward) when the desired physiological response is obtained, whereas it is negative when the undesired physiological response occurs. Thus, the patient learns to control his or her own physiological process (Figure 3).

When the physiological signals of interest are extracted from EEG, this technique is called EEG biofeedback, neurofeedback, or neurotherapy. The electrophysiological signals are believed to be related to different functional and mental states. The patient can hear or see an audio or visual positive feedback whenever the target parameters equal or exceed a threshold setting. The threshold is usually adjusted periodically to ensure the patient can receive the feedback over a fixed duration of time. The patient is instructed to discover the mental set or strategy to produce and maintain the positive feedback; no further instruction is given. The sessions are discontinued when the patient reaches the desired level of brain activity and/or behavioral improvement, when the neurophysiologic and/or neuropsychological outcomes remain stable, or after a fixed number of sessions.

Originally, the target electrophysiological signal in EEG biofeedback was the amplitude in a given frequency band, and the purpose was to normalize the EEG by increasing abnormally weak frequency bands and/or decreasing excessively dominant frequency bands. However, other physiological parameters can be targeted. Thornton (49) described 2 distinct categories. In addition to the absolute magnitude in a given frequency band, the activation measures are comprised of the 
relative magnitude (ratio of the magnitude of one band to the total magnitude of all bands), peak amplitude, peak frequency, and symmetry (peak amplitude symmetry between 2 locations in a particular bandwidth). The connection measures are mainly comprised of measures of coherence and phase.

The promise of EEG biofeedback is to promote normalization of abnormal brain activity and thus lead to behavioral and cognitive advantages. This promise is not specific to TBI, and indeed, EEG biofeedback is explored and claimed to be of benefit in a long list of diverse conditions, reaching from anxiety/mood disorders, attention deficit and hyperactivity disorders and autism, to age-related cognitive decline, and dementia. This chapter focuses on the notion that EEG biofeedback might leverage the diagnostic virtues of quantitative EEG in TBI and offer a valuable therapeutic intervention.

Two main approaches of EEG biofeedback in patients with TBI can be found in the literature. The first one relies on predefined protocols, based on previous studies revealing EEG abnormalities in patients with TBI, aiming to eliminate supposed abnormalities. The second one is based on individual deviations from normal EEG values as defined by a control group of participants or with a previously constituted database. Although most protocols train patients to control their brain activity at rest (eyes opened or eyes closed conditions), rehabilitating the EEG abnormalities while the patient is performing a task involving the target function is also possible and might increase the efficiency of EEG biofeedback.

According to the aim of the protocol, the EEG electrodes of interest can differ. By default, the vertex of the head (electrode position $\mathrm{Cz}$ ) is generally chosen. However, in TBI, one can choose the electrode closest to the impact site of the head injury (e.g., 50) or electrodes that reveal the largest abnormalities (e.g., 51). The frequencies considered have been traditionally limited to frequencies lower than 32 Hz. Higher frequencies (high beta or gamma bands), nevertheless, may also have multiple functions in sensory and cognitive processing and are of interest for the rehabilitation of patients with TBI (52). It should be noted that different definitions of the frequency bands are given across different studies; moreover, methods of calculation of different parameters (e.g., coherence) might vary from one study to another. Thus, generalization of any results in this field requires special care.

The single-case study of Byers (50) offers an example of a protocol aiming to adjust the level of activity in predefined frequency bands. A patient who sustained a TBI 6 years earlier was trained to enhance, over the $\mathrm{Cz}$ location, his or her sensory motor rhythm $(12-15 \mathrm{~Hz})$ and in a second time his or her beta activity $(15-18 \mathrm{~Hz})$ while at the same time suppressing theta activity $(4-7 \mathrm{~Hz})$. The expected modifications of frequency were not clearly obtained; nonetheless, many symptoms of this patient were reduced during and following the EEG biofeedback training. The improvement was mainly seen in cognitive flexibility and executive functions.

An example of connectivity training at rest, to normalize coherence values toward 
values measured in a group of healthy subjects, can be found in the study of Walker et al. (51). Twenty-six patients with TBI with symptoms interfering with daily activities for more than 3 months, including employment, were trained to increase their reduced coherence values and to decrease any elevated coherence values. The initial training involved the use of a pair of electrodes with the most significant abnormalities. After 5 sessions, the training was dedicated to the next pair of electrodes picking up the most significant abnormalities and so on. Using a global improvement scale, based on a reduction of symptoms (e.g., headaches, memory loss or confusion) and the ability to return to work, significant improvements were noted in $88 \%$ of the patients.

The studies from Tinius and Tinius (53), Thornton (49), and Thornton and Carmody (52) exemplify EEG biofeedback training while the patient is performing cognitive training or a task involving the function to be improved. In the study of Tinius and Tinius (53), the treatment decisions for 16 patients with mild TBI followed preestablished rules based on clinical symptoms and a brain map from the Thatcher reference database. For example, if theta activity was high, the treatment aimed to decrease theta activity at $\mathrm{Cz}$; if the primary symptom was pain, the target was to increase sensory motor rhythm at $\mathrm{Cz}$. They postulated that it may be beneficial to use coherence training after unipolar training and that it should start with the rehabilitation of short connections before long connections. Following this methodology, patients reported a decrease in their symptoms, and there was improvement of visual and auditory sustained attention that was trained during the simultaneous cognitive tasks. For the rehabilitation of memory function in patients with TBI, Thornton (49) offered a database for normal EEG reference during 18 different tasks (obtained from subjects without neurological disorder, history of brain injury or learning problems). The EEG variables were correlated with the memory performance of 59 normal right-handed participants to determine the cortically based electrophysiological correlates of effective cognitive functioning. Then, in several multiple single-case studies $(49,52)$, patients with TBI were trained to normalize abnormal connections (coherence and phase values), and this was associated with improvement of general cognitive abilities and memory function.

In summary, EEG biofeedback in patients with TBI is a promising field. However, it needs to be further explored. Thornton and Carmody (54) point out the heterogeneity of parameters chosen and outcomes measured in studies of EEG biofeedback in TBI. It remains to be systematically proven that $(a)$ abnormal EEG parameters and behavioral deficits are correlated, $(b)$ EEG biofeedback is effective in normalizing EEG, (c) EEG biofeedback is effective in improving behavior and cognition, $(d)$ measured improved functions translate into everyday life criteria, and (e) positive changes are long lasting. Ultimately, appropriately powered, randomized, controlled trials are needed.

In this context, a few questions are worth considering. Most prominently, how soon after a TBI the EEG biofeedback should be offered remains an open question. Starting too soon may overload existing resources, whereas waiting for a longer 
period of time may reduce the potential benefit (50-51). Several studies point toward the absence of a link between the time since the TBI and EEG abnormalities or successful outcomes of EEG biofeedback $(51,52,55)$. These observations contribute to the notion that the brain does not spontaneously repair the damage caused by the TBI but instead allocates different resources to accomplish the task with variable results (49).

\section{EVOKED POTENTIALS}

Following the presentation of a stimulus or multiple stimuli, an electrophysiological response from the nervous system is known as an EP. The stimuli are most frequently auditory, visual, or somatosensory, and the EPs are frequently recorded from the brain using EEG techniques. These potentials are different from conventional EEG because they are calculated from an averaged response to a presented stimulus. Such averaging allows the response to the stimuli to be isolated from the background EEG activity (Figure 4).

\section{Somatosensory Evoked Potential}

The somatosensory EP (SEP) captures the manner in which the neural system responds to sensory input. SEPs can be elicited through electrical, tactile, vibratory, or painful stimuli applied to different body parts. However, among these different modalities, electrical stimulation is most commonly employed because of its ease of use (56). A peripheral nerve, such as the median, ulnar, or tibial nerve, is stimulated, and the EP is picked up over the scalp (Figure 5). A SEP is generally characterized by its amplitude and latency. Short-latency SEPs (50 ms from stimulation) are more independent from the level of consciousness than longer latencies, which generally reflect higher cognitive processes. For example, absence of the N20 component of the SEPs, the component thought to mark the arrival of the thalamic volley to the cortex, appears to be a reliable indicator of significant cortico-subcortical disconnection and suggestive of poor prognosis. Overall, short-latency SEPs are considered valuable prognostic indicators for TBI (57).

In a consensus for the use of neurophysiologic techniques in TBI (58), short-latency EPs (including auditory and somatosensory) were found to be normal in $50 \%$ of severe TBI cases. Further, bilateral normal short-latency EPs predicted a favorable outcome in almost $80 \%$ of patients after TBI (59). However, a stronger prognostic indicator (albeit, negative) was in fact the absence of bilateral short-latency EPs (N20), and one study (57) showed a 95\% predictive value of not awakening from a coma with such a recording. A systematic review of 25 studies (60) showed that SEPs are the best single overall predictor of outcome after TBI, superior to CT, EEG, Glasgow Coma Scale (GCS), and pupillary and motor responses. However, when standard clinical tests such as GCS and pupillary and motor responses are combined with SEP recordings, the predictive ability is further enhanced.

Other EP studies such as brainstem auditory evoked potential (BAEP) are limited to 
evaluating pharmacological effects of hearing and brainstem dysfunction after brain injury $(56,61)$; visual evoked potential (VEP) to disturbances in the visual cortex. One study (62) combined the use of SEPs and BAEPs but noticed that it was really only SEPs that increased the predictive value of certain clinical parameters while neither of them correlated with cognitive function at 1-year follow-up.

Because EPs provide neurophysiologic monitoring of different neural pathways (SEPs somatosensory system, VEPs visual system, BAEPs auditory pathways, etc.), and because these various pathways show limited overlap, it seems reasonable to assume that multimodal EPs may be of additive diagnostic and prognostic value. A similar argument can be made regarding multimodal EPs within a given domain, where EPs are evoked with a variety of different stimuli (e.g., SEPs can be evoked by touch, pressure, electric stimuli, etc.) because these can tap onto different receptors and be mediated by different fiber pathways. Unfortunately, despite the theoretical appeal of such considerations, the practical use of such approaches is limited. Brain imaging techniques, particularly MRI, appear to offer greater clinical use.

In summary, the use of a SEP test can be of high value when assessing patients with brain injury and can add prognostic information to the clinical assessment. Certain situations need to be considered beforehand though, such as how the use of anesthesia can decrease the amplitude of SEP recordings, to prevent false conclusions.

\section{Event-Related Potential/P300}

An event-related potential (ERP) is an EP generally influenced by higher cognitive faculties. It is a measured brain response that is the result of either internal (e.g., thoughts) or external stimuli. Similar to EPs, ERPs are typically quantitatively characterized by their amplitude and latency. An ERP is usually referred to by its polarity (positive $[\mathrm{P}]$ or negative $[\mathrm{N}]$ ) and its latency in milliseconds.

A typical ERP protocol involves identifying and discriminating a specific stimulus in a larger series of stimuli (oddball paradigm). The target stimulus is generally presented $20 \%$ of the time, whereas the other stimuli (distractors) are presented $80 \%$ of the time (63). To accurately judge the brain's response to these stimuli, the experimenter must record multiple trials and then average the results. ERPs are thought to capture complex coordinated processing of widespread brain networks and appear to be a useful tool in assessing patients following brain injury because of their non-invasiveness and great temporal resolution (56).

One of the important elicited patterns of the ERP is the positive peak elicited $300 \mathrm{~ms}$ post-stimulus (P300) (63). This response is consistently observed whether the stimulus is visual, auditory, tactile, or even olfactory. It is thought to reflect active attention, working memory, and the ability to discriminate individual stimuli among a group of other similar stimuli (64-67). Following brain injury the absence of P300 does not necessarily predict a negative outcome (68). However, another study (69) 
emphasized the usefulness of visual ERPs for evaluation of abnormalities following trauma. Doi et al. (69) compared 20 patients with TBI with 32 age-matched controls using a conventional oddball paradigm. They found that the P300 latency was longer in patients than in the controls. In addition, the P300 amplitudes were significantly smaller in patients than in controls but only for certain stimuli. Thus, ERPs may be a potentially useful marker for evaluating cognitive dysfunction in patients after TBI. However, detailed attention to the type of stimulus is important, and the use of ERPs at individual (rather than group) level is insufficiently studied.

Abnormalities in ERP have also been found in asymptomatic patients with TBI. For instance, a 3-tone auditory oddball paradigm revealed subclinical deficits in concussed athletes (e.g., 70-71). This paradigm consists of 3 different stimuli presented in a random order: typically a standard tone presented in $80 \%$, a deviant target tone presented in $10 \%$, and a deviant non-target tone presented in $10 \%$ of the trials. Participants are instructed to press a button when they hear the target stimulus while withholding their response to both standard and deviant non-target tones.

A P3a ERP is obtained by averaging brain responses to the rare deviant tone, whereas the P3b ERP is obtained by averaging brain responses to the rare target tone (Figure 5). Thus, the P3b component is analogous to the classic P300 described earlier. A P3b amplitude reduction is believed to reflect deficits in memory updating. The P3a component is thought to reflect frontal lobe function; reduced P3a amplitude and latency delays may reflect deficits in shifting of attentional resources to novel stimuli. Although concussed athletes generally show normal behavioral outcomes in the auditory (or the equivalent visual) oddball task, their P3a and/or P3b components frequently show reduced amplitude and/or an increased latency (71-73). Such abnormalities may resolve 2 years after the last multiple concussions (71), but one study showed abnormalities up to 3 decades after the last multiple concussions (70). Further studies along these lines, however, seem warranted. A reliable objective marker of brain function/dysfunction following TBI, such as this one, might provide valuable insights into the neurobiological impact of injury and the compensatory mechanisms that may render the patients asymptomatic but nevertheless render them vulnerable for long-term complications.

In summary, these ERP measures appear to represent a particularly sensitive tool to detect functional alterations unnoticed on classic neuropsychological tests. ERPidentified subclinical findings may explain the vulnerability of patients to subsequent concussions and the reported susceptibility of patients with TBI to develop long-term complications, including a progressive cognitive decline. Longitudinal studies using such measures seem to be warranted.

\section{TRANSCRANIAL MAGNETIC STIMULATION}

TMS is a noninvasive method that uses the principles of electromagnetic induction 
to induce currents within discrete brain regions $(74,75)$. These currents can be of sufficient magnitude to depolarize neurons. When applied repetitively, TMS can modulate cortical excitability, decreasing or increasing it, depending on the parameters of stimulation, beyond the duration of the train of stimulation.

Following single-pulse TMS, distinct episodes of enhanced and suppressed activity can be observed. Initial induction of an excitatory postsynaptic potential is followed by a period of suppression of 100-200 ms duration. Furthermore, local and distant reentry mechanisms contribute to complex and longer lasting suppressionactivation dynamics. This results in lasting neuromodulation with a complex pattern of suppression and facilitation of activity, in part related to stimulation of inhibitory and excitatory interneurons, metabotropic or metabolic processes, or even vascular responses.

TMS represents a particularly pertinent approach suitable to study the neurophysiologic effects of TBI because of its unprecedented sensitivity to central excitation/inhibition (E/I) mechanisms (76). TMS can provide additional tools to characterize severity of TBI and evaluate abnormalities in symptomatic and asymptomatic patients. Moreover, the ability to induce plasticity with TMS can provide an interventional tool to promote recovery.

\section{Characterization of Brain Abnormalities After TBI: Single-and Paired-Pulse TMS}

EP approaches can be readily adapted along with the use of TMS. TMS is applied as a controlled input to a specific brain region, and the neurophysiologic nervous system response can be recorded using electromyography (EMG) or EEG. The most commonly used EPs elicited with TMS are motor-evoked potentials (MEPs). They are produced by using TMS to target the primary motor cortex (M1) and are recorded using EMG via electrodes placed over specific target muscles. MEPs are of great interest to evaluate corticospinal integrity in patients with TBI.

\section{Central Motor Conduction Time}

Central motor conduction time (CMCT) reflects the integrity of the corticospinal tract (77). It is calculated by subtracting the peripheral conduction time (spinal cord to muscles) from the latency of MEPs evoked by TMS. CMCT has been shown to be prolonged in patients with TBI with diffuse and combined brain lesions tested 2 weeks after head trauma (78). However, CMCT was not affected in patients with TBI with minor brain concussions or focal lesions (78). Nonetheless, this absence of CMCT increase does not necessarily demonstrate an absence of impairment at the cortical level.

\section{Resting Motor Threshold and Motor-Evoked Potentials Amplitude}

Resting motor threshold (RMT) refers to the lowest TMS intensity necessary to 
evoke MEPs in a target muscle when single-pulse stimuli are applied to the contralateral M1. RMT reflects neuronal membrane excitability, which is highly dependent on ion channel conductivity (79-80). When TMS is applied at suprathreshold intensities, activation of excitatory interneurons results in volleys of upper motor neuron activity, which subsequently activate motor neurons in the spinal cord. The summed activity results in an MEP. Latency and peak-to-peak amplitude reflect the integrity of the corticospinal motor pathways. The MEP/M wave amplitude ratio is calculated by dividing the MEP amplitude by the maximal M wave amplitude obtained after supramaximal peripheral electrical stimulation.

RMT did not reveal any abnormality, neither in athletes with 1 to several concussions 9 months after their last concussion (81) nor in athletes with a history of sports concussions more than 30 years prior to testing (70). However, in another study, RMT was significantly increased 2 weeks after mild and moderate head injury (78). This increase was accompanied by a marked reduction in the MEP/M wave amplitude ratio. Similarly, concussed athletes evaluated sequentially between 1 and 10 days post-concussion showed a progressive increase in MEP latency and a reduction in MEP amplitude (82). The loss of the corticospinal neurons, the slowing because of demyelination or axonal disconnection, and the desynchronization of multiple descending volleys resulting in less effective temporal summation of excitatory postsynaptic potentials could explain these observations $(78,82)$. In addition, the reduction of the MEP amplitude may also be indicative of pyramidal tract/brainstem involvement (82).

Finally, increased RMT was also found 3 months after mild-to-moderate TBI in patients with objective excessive daytime sleepiness (83). A reduced excitability of the corticospinal system during wakefulness, mimicking the hyperpolarization of the thalamocortical system in healthy subjects during sleep, might contribute to the persistent sleepiness often seen in patients with TBI.

\section{Cortical Silent Period}

When TMS is delivered over the motor cortex while the subject maintains a voluntary muscle contraction in the contralateral hand, a pause in ongoing EMG activities follows the MEP (Figure 6D). This pause is called the cortical silent period or contralateral silent period (CSP). The initial phase of the CSP might be related to the refractory period of the pyramidal tract neurons, whereas the latter part of the CSP has been attributed to activity of intracortical inhibitory systems of the M1 involving gamma-aminobutyric acid $\left(\mathrm{GABA}_{\mathrm{B}}\right)$ receptors.

De Beaumont et al. (81) and Tremblay et al. (84) showed that CSP duration was prolonged in athletes who had experienced multiple concussions. Sustaining subsequent concussions exacerbates this deficit and thus provides additional support for the existence of cumulative deficit following multiple concussions; moreover, concussion severity was significantly correlated with CSP lengthening 
(81). Observed CSP duration lengthening in athletes with multiple concussion seemed to remain unaffected by the time elapsed since the last accident. De Beaumont et al. (70) further showed that former athletes with a history of concussion more than 30 years prior to testing also have an increased CSP duration, despite apparently normal cognitive performance and absence of neuropsychological abnormalities.

However, a previous study (78) showed no alteration of CSP duration in a similar population of patients 2 weeks after a mild TBI; only patients with moderate brain injury showed an increased CSP duration. Such discrepancy could be related to methodological aspects in relation to determination of TMS intensity (78). Alternatively, CSP prolongation could be triggered later after trauma, when acute RMT abnormalities are resolved (81).

\section{Ipsilateral Silent Period}

When TMS is applied over M1 during an ongoing tonic voluntary contraction of the muscles ipsilateral to the site of stimulation, the activity of these ipsilateral muscles can be temporarily suppressed. This ipsilateral silent period (ISP) has been attributed to transcallosal inhibition, and this method can evaluate the integrity of the corpus callosum connecting homologous motor cortices. Diffuse axonal injury, in consequence of TBI, might involve disruption of the corpus callosum, which may be uncovered as a reduction of transcallosal inhibition measured with TMS. Takeuchi et al. (85) showed that the amount of transcallosal inhibition was significantly reduced in patients with TBI several months after their concussion compared to healthy controls, and this reduction was significantly correlated with the severity of TBI as evaluated using the GCS.

Transcallosal inhibition can also be assessed by measuring the decrease in amplitude of an MEP evoked by a test pulse applied over the contralateral M1 when this test pulse is preceded by a conditioning pulse applied over the ipsilateral M1 (dual-coil paired-pulse TMS technique). This paired-pulse technique using 2 TMS coils has not yet been used in a TBI population but offers promise to further characterize possible interhemispheric and other corticocortical disconnections.

\section{Excitatory and Inhibitory Balance}

Chistyakov et al. (78) evaluated the balance between excitatory and inhibitory central mechanism by calculating the interthreshold difference (ITD) as the difference between the RMT and CSP threshold. Indeed, both RMT and CSP thresholds were significantly increased in patients who sustained mild and moderate head injury, but the increase in CSP threshold was much less pronounced than that of the MEP threshold. This resulted in a significant increase of the ITD. The increase in the ITD, accompanied by reduction of the MEP/M wave amplitude ratio, suggests dissociated impairment of inhibitory and excitatory components of the central motor control. 
Alternative ways to assess excitatory and inhibitory central mechanisms is using paired-pulse TMS (ppTMS) paradigms. The ppTMS involves the application of 2 TMS stimuli of independently controllable intensity and with a variable interstimulus interval to the same cortical region. The first stimulus, thus, serves as a conditioning stimulus to the effect of the second test stimulus. Several paired-pulse paradigms have been designed to assess the short-interval intracortical inhibition (SICI; Figure $6 \mathrm{~B})$, hypothetically $\mathrm{GABA}_{\mathrm{A}}$ mediated, the long-interval intracortical inhibition (LICI), hypothetically $\mathrm{GABA}_{\mathrm{B}}$-mediated, and intracortical facilitation (ICF; Figure $6 C)$, hypothetically mediated by synaptic glutamatergic transmission $(80,86-88)$.

The studies from De Beaumont and collaborators previously cited $(70,81,84)$ did not reveal any abnormalities in SICI or ICF in athletes with 1 or several sport concussions from 9 months to 30 years prior to testing. However, increased SICI has been found 3 months after mild-to-moderate TBI in patients with objective excessive daytime sleepiness (83). These patients also had an increased RMT. Both RMT and SICI correlated with objective measures of sleepiness. It has been suggested that the persistent sleepiness in some patients with TBI is caused by a combination of reduced excitability because of reduced hypocretin signaling (hypothalamic injury) and also injury to other sleep-wake regulating systems.

Finally, LICI was enhanced in asymptomatic concussed athletes with multiple concussions that occurred more than 12 months prior to testing (84). Together with the increase in CSP duration, this result points toward the presence of specific and stable alterations of $\mathrm{GABA}_{\mathrm{B}}$ receptor activity in the M1 (84).

In summary, TMS methodology can provide useful tools to assess brain abnormalities in the acute and sustained phases of TBI of various severities. Acutely after moderate concussion, several measures point toward the loss of the corticospinal neurons, the slowing and the desynchronization of multiple descending volleys, and both excitatory and inhibitory circuits seem to be affected. This could be related to cholinergic abnormalities and excessive glutamate accumulation leading to $N$-methyl-D-aspartate (NMDA)-mediated excitotoxicity. Whether these deficits are also present in asymptomatic patients with mild TBI, shortly after the concussion, remains to be explored. In the long term $(9$ months and up to 30 years after the concussion), potential glutamatergic excitotoxicity of asymptomatic patients with mild TBI seems to resolved (normal excitability and ICF), and there is likely no deficit in $\mathrm{GABA}_{\mathrm{A}}$-mediated inhibition (normal SICI). However, an increase in CSP duration and an abnormal LICI point toward increased $\mathrm{GABA}_{\mathrm{B}}$ transmission. It has been suggested that this increase may counter preliminary excitotoxicity and prevent damage; however, it might be excessive and finally maladaptive (84). Whether similar mechanisms occur in the sustained phase of patients with more severe TBI remain to be explored.

TMS studies are of particular importance to further characterize the severity of TBI and evaluate subclinical lasting effects. They can also provide insight into the 
mechanisms of symptoms associated with TBI, such as sleepiness, and help to identify patients who would be suitable for treatment (83). If further developed, TMS tools could be included in the return-to-normal-life criteria. They might turn out to be useful in the prognostic concerning recovery and improve prognosis of a second TBI or the development of mild cognitive impairment or Alzheimer disease (84).

\section{TMS Combined with EEG}

The combination of brain stimulation by TMS with simultaneous EEG recording has become feasible because of the development of novel engineering solutions (89-92). The TMS-EEG integration provides real-time information on cortical reactivity and connectivity. A noninvasive input (TMS) of known spatial and temporal characteristics can be applied to study local reactivity of the brain and interactions between different brain regions with directional and precise chronometric information (Figure 7). TMS-EEG combination appears to be of particular interest to explore excitability of areas outside the motor cortex that are primarily affected by TBI. In addition, this methodology will allow one to assess the integrity of entire cortical circuits. Being able to study humans directly and across the lifespan is critical to translate findings from animal studies and thus identify potential biomarkers for disease and promote therapeutic monitoring. Systematic exploration of such TMS-EEG methods in TBI seems warranted and will allow the study of prefrontal, temporal, and other brain regions and distributed neural networks that cannot be approached using TMS-EMG techniques.

\section{Repetitive TMS}

Trains of repeated TMS (rTMS) pulses can induce a lasting modification of activity in the targeted brain region, which can outlast the effects of the stimulation itself. Depending on the frequency, intensity, and the pattern of stimulation, the induced effects promote inhibition or excitation of the stimulated area. Repetitive TMS paradigms might be used to test the plasticity resources of patients at several time points after TBI of varying severities. Indeed, after a TBI, the nervous system reorganizes in response to injury. Such reorganization is restricted by existing patterns of anatomical and functional brain connectivity. The behavioral impact of such plastic reorganization is not necessarily adaptive and may prove to represent dead-end strategies that ultimately limit functional recovery and promote lasting disability. Assessment of plasticity resources at different time points after TBI might be necessary to develop differential mechanistic interventions and promote functional recovery.

In addition, rTMS can be directly used to facilitate recovery. Such rTMS approaches thus offer, similar to EEG biofeeback, the opportunity to use neurophysiologic techniques in therapeutic rather than diagnostic and prognostic applications. Pape et al. (93) performed 30 sessions (6 weeks) of an rTMS protocol in a patient with severe TBI who remained in a vegetative state for longer than 9 months. The rTMS 
intervention (a repetitive paired-pulse stimulation of the right dorsolateral prefrontal cortex) was designed to use potentially excitatory stimulation parameters while maximizing safety. This methodology proved to be safe, and the patient progressed clinically from a vegetative state at the time of study enrollment to a minimal conscious state by the 15th session. The patient demonstrated incremental neuro-behavioral improvements simultaneously occurring with the provision of rTMS up to the 25th session. Although a mild decrease of performance occurred during the final 5 sessions, most of the neurobehavioral improvement sustained 6 weeks after rTMS withdrawal and, according to his family, up to 1 year after completion of the study. Obviously, this is a single-case study that requires cautious follow-up and demands confirmation prior to clinical adoption. Whether rTMS might promote adaptive plasticity in other patients, including patients with less severe TBI, remains to be explored. Such studies should be done with care because rTMS can induce significant side effects and complications, particularly in certain predisposed populations (94). Therefore, appropriately controlled studies are needed prior to considering the use of rTMS in clinical practice.

\section{OTHER METHODS OF POTENTIAL INTEREST}

Transcranial direct current stimulation (tDCS) is a noninvasive technique of neuromodulation, which passes low amplitude direct current (1-2 mA) through pad electrodes placed on the scalp to alter neuronal firing. Although anodal tDCS elicits prolonged increases in the cortical excitability of the underlying brain area, cathodal stimulation shows opposite effects (95-96). As with rTMS, the duration of the effects outlast the period of stimulation. The mechanisms are believed to be nonsynaptic and result from change in resting polarization of neurons. Although investigation of tDCS in patients with TBI is only starting, this technique is promising because it shows excellent safety record and has proven to be able to improve several brain functions in healthy subjects and in patient populations (for a review, see 97). Several studies conducted on the safety of tDCS have concluded that it is a painless technique for electrically stimulating the brain with almost no risk of harm. The most frequent adverse effects that have been reported include moderate fatigue $(35 \%)$, mild headache $(11.8 \%)$, nausea $(2.9 \%)$ and temporary mild tingling sensation, itchiness, and/or redness in the area

of stimulation. Overall, tDCS features a highly portable, safe, noninvasive means to modulate cortical excitability with reasonable topographic resolution and reliable experimental blinding. It can focally suppress or enhance neuronal firing following TBI and thus may offer a promising method to minimize the damage and promote functional recovery. Cathodal tDCS may be employed to suppress the acute glutamatergic hyperexcitability following TBI. In the subacute stage, when GABAergic activity is excessive and conditions the neurologic, cognitive, and functional disability, anodal tDCS may increase excitability to counter these aberrant GABAergic effects. In the chronic stage, brain stimulation coupled to rehabilitation can enhance behavioral recovery, learning of new skills, and cortical plasticity. Furthermore, tDCS can be combined, with relative ease, with other 
interventions with the aim of enhancing their effect. For example, tDCS can be applied during cognitive training, robot-supported arm or gait training, physical or occupational therapy, imagery, and so forth. As such, tDCS might prove to be a valuable neuromodulatory tool to promote rehabilitation and functional recovery after TBI.

Cranial electrotherapy/electrical stimulation (CES) is a technique that provides small pulses of electric current (0-4 mA) across the head, using pre-gelled electrodes, conductive rubber ear clips, or moistened sponges placed on the head either below or directly on the ears. This technique has primarily been investigated for the treatment of anxiety, depression, and insomnia. It is hypothesized that the outcomes of CES would be mediated by neurotransmitters. Some studies have shown effects onto QEEG. However, the mechanisms of action remain uncertain, and overall, the experimental evidence regarding clinical use is limited. In the treatment of post-concussion symptoms, CES has been shown to be useful in improving several mood measures (98), but further studies with better controlled trial designs are needed to assess its efficacy.

Transcranial pulsed ultrasound stimulation (TCPUS) is another recently developed method of potential interest for noninvasive brain stimulation (99-100). Ultrasound is a mechanical pressure wave with a frequency above the range of human hearing ( $20 \mathrm{kHz}$ ), capable of being transmitted over long distances through solid structures. Ultrasound can influence physiological activity through thermal and/or mechanical mechanisms. Tufail et al. (99) used a series of low-frequency ultrasound pulses (typically below 100 cycles per pulse at a frequency within onetenths of a $\mathrm{MHz}$ range) repeated over time (typically hundreds of times at a frequency in the order of $1 \mathrm{KHz})$ at a low intensity $(<300 \mathrm{~mW} / \mathrm{cm} 2)$ to safely stimulate neural activity in the intact mouse brain. With a negligible increase of temperature, ultrasound stimulation of the motor cortex produced short bursts of activity and peripheral muscle contraction, whereas stimulation of the hippocampus triggered rhythmic bursting lasting about 3 seconds. It was suggested that the fluidmechanical effects $(a)$ modulate the resting membrane potentials of neurons, $(b)$ directly modulate the kinetics of mechanically sensitive ions channels, and/or $(c)$ produce ephaptic effects by altering the distribution of electric fields. The advantage of this technique over other noninvasive techniques of brain stimulation would be its spatial resolution, estimated to be of approximately $2 \mathrm{~mm}$. Although this technique appears to be safe in mice, it remains to be proven as to whether it could be safely applied in other species.

Transcranial Doppler sonography (TCD) can be used in the acute assessment of cerebral ischemia following TBI (101) and may offer appealing noninvasive methods to monitor patients in the intensive care setting. A study in patients with mild-to-moderate TBI (102) showed that TCD-based measures of brain perfusion at time of hospitalization are good predictors of overall neurological outcomes. Thus, TCD might be a powerful prognostic tool in TBI. More recent advances in our understanding of TCD have lead to exciting possible therapeutic roles in TBI where 
clot formation may result from primary injury. One application is sonothrombolysis, a technique of focal TCD applied at diagnostic frequencies alone or in combination with standard thrombolytic therapy (tPA) (103). TCD may also have the potential to promote neuroprotection during acute TBI by increasing the local bioavailability of neuroprotective agents. TCD has been shown to transiently (i.e., hours) enhance blood-brain barrier (BBB) permeability without adverse cellular effects. The mechanism is believed to be a process of stable cavitation in which low acoustic energy causes administered microbubbles to oscillate and expand creating small eddy currents in the surrounding plasma. These currents provide shear stress on cells and large molecules to improve BBB transcellular and paracellular transport (104). Thus, TCD may increase the applicability of novel neuroprotective agents by allowing focal pharmacokinetic optimization. Finally, some studies have alluded to the potential of TCD as a direct means of neuroprotection (105). In the setting of TBI, these attributes of TCD may allow for suppression of neuronal activity in the acute energy deficient phase and facilitation in the subacute phase of active recovery, strengthening our therapeutic capacity against TBI.

Low-level laser therapy (LLLT), or photobiostimulation, is a novel method of noninvasive neural stimulation that, at specific wavelengths, can safely penetrate into the brain. LLLT is thought to promote cellular survival in times of reduced energy substrate through interactions with cytochrome $\mathrm{c}$ oxidase to enhance oxidative phosphorylation, improve mitochondrial function, and increases adenosine triphospate (ATP) $(106,107)$. LLLT has been shown to accelerate wound healing, reduce neurological deficit following stroke, and improve outcome in spinal cord injury. Recently, LLLT was used for the first time in a rodent TBI model. LLLTtreated rats showed significantly reduced functional impairment and reduced lesion volume (108). Given such preclinical findings, the promising results in human stroke research (109-111), and the unique properties of LLLT, its therapeutic application in TBI seems worth exploring.

\section{SOME LIMITATIONS OF ELECTROPHYSIOLOGICAL TECHNIQUES}

Although all these techniques have proven to be helpful for TBI diagnosis, prognosis, exploration of the mechanisms, and/or rehabilitation, several concerns still need to be addressed. The reliability of most of these measures is particularly challenged by the fact that the dissociation of TBI or non-TBI is often complicated by ongoing or comorbid conditions and medications.

QEEG allows successful discrimination between mild TBI and controls of patients with more severe TBI. EPs can provide valuable prognostic information and ERPs and brain stimulation can further contribute to fine characterization of brain dysfunction in TBI. However, research is still needed to assess the impact of potential confounding factors on most of these techniques. For example, prior conditions, such as neurological or psychiatric issues, may affect the outcomes of all the previously mentioned techniques. In addition, it is difficult to disentangle neurophysiologic abnormalities as a consequence to the TBI per se from conditions 
that are often associated with it, such as PTSD, depression, or anxiety.

Moreover, it remains to be fully explored how medications systematically affect the normative data bases used for all the techniques described in this chapter. Among the medications relevant for TBI, anesthetics, analgesics, and antiepileptic drugs are particularly prone to modify neurophysiologic measures. Finally, often leading to a TBI event, ingestion of alcohol and toxins should be discussed prior to interpretation of EEG findings and/or application of brain stimulation. In fact, drugs and alcohol could alter brain excitability and increase the risk of adverse events associated with TMS and other stimulation techniques. Potential sleep deprivation, occurring as a consequence of TBI-related insomnia, also has to be considered in the same context. In addition, increased intracranial pressure, intracranial lesion or hematomas, and structural damage might all constitute contraindication for TMS and other brain stimulation methods because they might be aggravated by stimulation. Cranioplasty and burr holes might participate in a shunting on the induced current through skull defects, leading to poorly controlled intensity of stimulation in brain tissues. Finally, even in patients with mild TBI, where none of these complications are observed, safety studies remains to be undertaken for most brain stimulation methods.

\section{CONCLUSION}

Electrophysiological techniques are essential in the TBI care practice. They are useful for diagnosis, prognosis and monitoring, for exploration and characterization of brain deficits, and for detection of subclinical anomalies that might increase vulnerability to subsequent concussions or susceptibility to long-term complications. In addition, some of these tools can be used to guide functional recovery (e.g., EEG biofeedback, brain stimulation). Certainly, these techniques still need to be developed and improved, and for all, studies need to be done to further clarify findings and to assess the validity and safety of these methods when confounding and aggravating factors intervene. 


\section{KEY CLINICAL POINTS}

1. The use of conventional EEG is limited for mild TBI. However, for more severe TBI, conventional EEG is useful for prognosis, monitoring the recovery/deterioration, detecting brain damages, or post-traumatic epilepsy.

2. QEEG allows a more objective characterization of brain abnormalities after TBI and appears sensitive to discriminate mild TBI from healthy controls and from more severe TBI. Efforts still need to be made to reconcile results from different studies and establish the full clinical use of QEEG.

3. Based on the findings of EEG anomalies, EEG biofeedback is a promising potential treatment for patients with TBI, aiming to normalize the EEG and consequently improving behavior and cognition.

4. SEPs and ERPs are useful together with other clinical assessments for prognosis of TBI, but their interpretation remains delicate. They are also sensitive tools to detect functional alterations and may help in the understanding of vulnerability to subsequent concussions as well as the susceptibility to develop long-term complications.

5. Methods of brain stimulation, such as TMS, are particularly suited to assess excitatory and inhibitory function in patients with TBI. Combined with EEG and also neuroimaging, noninvasive brain stimulation might become a particularly useful physiologic biomarker, but more work is needed.

6. Brain stimulation applied for neuromodulation (for example, repetitive TMS or tDCS) is also promising as a tool to guide functional recovery and adaptive plasticity. Safety guidelines for the TBI populations still need to be established.

\section{KEY REFERENCES}

1. De Beaumont L, Théoret $H$, Mongeon $D$, et al. Brain function decline in healthy retired athletes who sustained their last sports concussion in early adulthood. Brain. 2009; 132(pt 3):695 - 708.

2. Louise-Bender Pape T, Rosenow J, Lewis G, et al. Repetitive transcranial magnetic stimulation-associated neuro-behavioral gains during coma recovery. Brain Stimul. 2009;2(1):22 - 35.

3. Robinson LR, Micklesen PJ, Tirschwell DL, Lew HL. Predictive value of somatosensory evoked potentials for awakening from coma. Crit Care Med. 2003;31(3):960-967.

4. Thatcher RW. Electroencephalography and mild traumatic brain injury. In: Slobounov S, Sebastianelli W, eds. Foundations of Sport-Related Brain Injury. New York, NY: Springer-Verlag; 2006:241-265.

5. Thornton KE, Carmody DP. Efficacy of traumatic brain injury rehabilitation: interventions of QEEG-guided biofeedback, computers, strategies, and medications. Appl Psychophysiol Biofeedback. 2008;33(2):101-124. 


\section{REFERENCES}

1. Tatum WO IV, Husain AM, Benbadis SR, Kaplan PW. Normal adult EEG and patterns of uncertain significance. J Clin Neurophysiol. 2006;23(3):194-207.

2. Kaiser J, Lutzenberger W. Human gamma-band activity: a window to cognitive processing. Neuroreport. 2005;16(3):207-211.

3. Arciniegas DB. Clinical electrophysiologic assessments and mild traumatic brain injury: state-of-the-science and implications for clinical practice. Int J Psychophysiol. 2011;82(1):41-52.

4. Thatcher RW, North DM, Curtin RT, et al. (2001). An EEG severity index of traumatic brain injury. J Neuropsychiatry Clin Neurosci. 2011;13(1):77-87.

5. Steudel WI, Kruger J. Using the spectral analysis of the EEG for prognosis of severe brain injuries in the first post-traumatic week. Acta Neurochir Suppl (Wien). 1979;28(1):40-42.

6. Wallace BE, Wagner AK, Wagner EP, McDeavitt JT. A history and review of quantitative electroencephalography in traumatic brain injury. J Head Trauma Rehabil. 2001;16(2):165-190.

7. Abend NS, Dlugos DJ, Hahn CD, Hirsch LJ, Herman ST. Use of EEG monitoring and management of non-convulsive seizures in critically ill patients: a survey of neurologists. Neurocrit Care. 2010; 12(3):382 - 389.

8. Nuwer MR, Hovda DA, Schrader LM, Vespa PM. Routine and quantitative EEG in mild traumatic brain injury. Clin Neurophysiol. 2005;116(9):2001-2025.

9. Hughes JR, John ER. Conventional and quantitative electroencephalography in psychiatry. J Neuropsychiatry Clin Neurosci. 1999; 11(2):190-208.

10. Jacome DE, Risko M. EEG features in post-traumatic syndrome. Clin Electroencephalogr. 1984;15(4):214-221.

11. Voller B, Benke $T$, Benedetto $K$, Schnider $\mathrm{P}$, Auff E, Aichner F. Neuropsychological, MRI and EEG findings after very mild traumatic brain injury. Brain Inj. 1999;13(10):821-827.

12. Denker PG, Perry GF. Postconcussion syndrome in compensation and litigation; analysis of 95 cases with electroencephalographic correlation. Neurology. 1954;4(12):912-918.

13. Jacome DE. EEG in whiplash: a reappraisal. Clin Electroencephalogr. 1987;18(1):41 - 45 .

14. Torres F, Shapiro SK. Electroencephalograms in whiplash injury. A comparison of electroencephalographic abnormalities with those present in 
closed head injuries. Arch Neurol. 1961;5:28-35.

15. Dow RS, Ulett G, Raaf J. Electroencephalographic studies immediately following head injury. Am J Psychiatry. 1944;101:174-183.

16. Courjon J, Scherzer E. Traumatic disorders. In: Remond A, Magnus O, Courjon J, eds. Handbook of Electroencephalography and Clinical Neurophysiology: Clinical EEG, IV. Amsterdam, The Netherlands: Elsevier; 1972:1-104.

17. Fenton GW. The postconcussional syndrome reappraised. Clin Electroencephalogr. 1996;27(4):174-182.

18. Geets W, de Zegher F. EEG and brainstem abnormalities after cerebral concussion. Short term observations. Acta Neurol Belg. 1985; 85(5):277 283.

19. Geets W, Louette N. EEG and brain-stem evoked potentials in 125 recent concussions [in French]. Rev Electroencephalogr Neurophysiol Clin. 1983;13(3):253-258.

20. Hessen E, Nestvold K. Indicators of complicated mild TBI predict MMPI-2 scores after 23 years. Brain Inj. 2009;23(3):234-242.

21. Koufen H, Dichgans J. Frequency and course of posttraumatic EEGabnormalities and their correlations with clinical symptoms: a systematic follow up study in 344 adults (author's transl) [in German]. Fortschr Neurol Psychiatr Grenzgeb. 1978;46(4):165-177.

22. Arfel G. Introduction to clinical and EEG studies in coma. In: Remond A, Harner R, Naquet R, eds. Handbook of Electroencephalography and Clinical Neurophysiology: Clinical EEG, II. Amsterdam, The Netherlands: Elsevier; 1972:5-23.

23. Bricolo A, Turella G. Electroencephalographic patterns of acute traumatic coma: diagnostic and prognostic value. J Neurosurg Sci. 1973;17:278 - 285.

24. Stone L, Keenan MA. Peripheral nerve injuries in the adult with traumatic brain injury. Clin Orthop Relat Res. 1988;(233):136-144.

25. Synek VM. Revised EEG coma scale in diffuse acute head injuries in adults. Clin Exp Neurol. 1990;27:99-111.

26. Synek VM. Prognostically important EEG coma patterns in diffuse anoxic and traumatic encephalopathies in adults. J Clin Neurophysiol. 1988;5(2):161174.

27. Hakkinen VK, Kaukinen S, Heikkila H. The correlation of EEG compressed spectral array to Glasgow Coma Scale in traumatic coma patients. Int J Clin Monit Comput. 1988;5(2):97 - 101. 
28. Rumpl E. Craniocerebral trauma. In: Niedermeyer E, da Silva FL, eds. Electroencephalography. 5th ed. Philadelphia, PA: Lippincott Williams \& Wilkins; 2005:415-438.

29. Rumpl E, Prugger M, Gerstenbrand F, Hackl JM, Pallua A. Central somatosensory conduction time and short latency somatosensory evoked potentials in post-traumatic coma. Electroencephalogr Clin Neurophysiol. 1983;56(6):583-596. 30.

30. Jabbari B, Vengrow MI, Salazar AM, Harper MG, Smutok MA, Amin D. Clinical and radiological correlates of EEG in the late phase of head injury: a study of 515 Vietnam veterans. Electroencephalogr Clin Neurophysiol. 1986;64(4):285-293.

31. Ruijs MB, Gabreels FJ, Thijssen HM. The utility of electroencephalography and cerebral computed tomography in children with mild and moderately severe closed head injuries. Neuropediatrics. 1994; 25(2):73 - 77.

32. Frey LC. Epidemiology of posttraumatic epilepsy: a critical review. Epilepsia. 2003;44(suppl 10):11-17.

33. Lewine JD, Davis JT, Bigler ED, et al. Objective documentation of traumatic brain injury subsequent to mild head trauma: multimodal brain imaging with MEG, SPECT, and MRI. J Head Trauma Rehabil. 2007;22(3):141-155.

34. Cervone RL, Blum AS. Normal variant EEG pattern. In: Blum AS, Rutkove SB, eds. The Clinical Neurophysiology Primer. Totowa, NJ: Humana Press; 2007:83-100.

35. So EL. Interictal epileptiform discharges in persons without a history of seizures: what do they mean? J Clin Neurophysiol. 2010;27(4): 229 - 238.

36. Malow BA. Sleep deprivation and epilepsy. Epilepsy Curr. 2004; 4(5):193 195.

37. Thomaides TN, Kerezoudi EP, Chaudhuri KR, Cheropoulos C. Study of EEGs following 24-hour sleep deprivation in patients with posttraumatic epilepsy. Eur Neurol. 1992;32(2):79-82.

38. Benbadis SR, O'Neill E, Tatum WO, Heriaud L. Outcome of prolonged videoEEG monitoring at a typical referral epilepsy center. Epilepsia. 2004;45(9):1150-1153.

39. Arciniegas DB, Anderson CA, Topkoff J, McAllister TW. Mild traumatic brain injury: a neuropsychiatric approach to diagnosis, evaluation, and treatment. Neuropsychiatr Dis Treat. 2005;1(4):311-327.

40. Leon-Carrion J, Martin-Rodriguez JF, Damas-Lopez J, Martin JM, Dominguez- 
Morales Mdel R. A QEEG index of level of functional dependence for people sustaining acquired brain injury: the Seville Independence Index (SINDI). Brain Inj. 2008;22(1):61-74.

41. Thatcher RW, Walker RA, Gerson I, Geisler FH. EEG discriminant analyses of mild head trauma. Electroencephalogr Clin Neurophysiol. 1989;73(2):94 106.

42. Kumar S, Rao SL, Chandramouli BA, Pillai SV. Reduction of functional brain connectivity in mild traumatic brain injury during working memory. $J$ Neurotrauma. 2009;26(5):665-675.

43. Thornton K. The electrophysiological effects of a brain injury on auditory memory functioning. The QEEG correlates of impaired memory. Arch Clin Neuropsychol. 2003;18(4):363-378.

44. Cao C, Slobounov S. Alteration of cortical functional connectivity as a result of traumatic brain injury revealed by graph theory, ICA, and sLORETA analyses of EEG signals. IEEE Trans Neural Syst Rehabil Eng. 2010;18(1):11-19.

45. Thatcher RW. Electroencephalography and mild traumatic brain injury. In: Slobounov S, Sebastianelli W, eds. Foundations of Sport-Related Brain Injury. New York, NY: Springer-Verlag; 2006:241-265.

46. Nuwer M. Assessment of digital EEG, quantitative EEG, and EEG brain mapping: report of the American Academy of Neurology and the American Clinical Neurophysiology Society. Neurology. 1997;49(1):277 - 292.

47. Hoffman DA, Lubar JF, Thatcher RW, et al. Limitations of the American Academy of Neurology and American Clinical Neurophysiology Society paper on QEEG. J Neuropsychiatry Clin Neurosci. 1999;11(3):401-407.

48. Thatcher RW, Moore N, John ER, Duffy F, Hughes JR, Krieger M. QEEG and traumatic brain injury: rebuttal of the American Academy of Neurology 1997 report by the EEG and Clinical Neuroscience Society. Clin Electroencephalogr. 1999;30(3):94-98.

49. Thornton KE. The improvement/rehabilitation of auditory memory functioning with EEG biofeedback. NeuroRehabilitation. 2002; 17(1):69 - 80.

50. Byers AP. Neurofeedback therapy for a mild head injury. J Neurotherapy. 1995;1(1):22 - 37.

51. Walker JE, Norman CA, Weber RK. Impact of qEEG-guided coherence training for patients with a mild closed head injury. J Neurotherapy. 2002;6(2):31 43.

52. Thornton KE, Carmody DP. Electroencephalogram biofeedback for reading 
disability and traumatic brain injury. Child Adolesc Psychiatr Clin N Am. 2005;14(1):137-162, vii.

53. Tinius TP, Tinius KA. Changes after EEG biofeedback and cognitive retraining in adults with mild traumatic brain injury and attention deficit hyperactivity disorder.J Neurotherapy. 2002;4(2):27-44.

54. Thornton KE, Carmody DP. Traumatic brain injury rehabilitation: QEEG biofeedback treatment protocols. Appl Psychophysiol Biofeedback. 2009;34(1):59 - 68.

55. Thornton KE, Carmody DP. Efficacy of traumatic brain injury rehabilitation: interventions of QEEG-guided biofeedback, computers, strategies, and medications. Appl Psychophysiol Biofeedback. 2008; 33(2):101 - 124.

56. Lew HL, Lee EH, Pan SSL, Chiang JYP. Electrophysiologic assesment techniques: evoked potentials and electroencephalography. In: Zasler ND, Katz DI, Zafonte RD, eds. Brain Injury Medicine. New York, NY: Demos Medical Publishing; 2007:157-165.

57. Robinson LR, Micklesen PJ, Tirschwell DL, Lew HL. Predictive value of somatosensory evoked potentials for awakening from coma. Crit Care Med. 2003;31(3):960-967.

58. Guérit JM, Amantini A, Amodio P, et al. Consensus on the use of neurophysiological tests in the intensive care unit (ICU): electroencephalogram (EEG), evoked potentials (EP), and electroneuromyography (ENMG). Neurophysiol Clin. 2009;39(2):71-83.

59. Amantini A, Grippo A, Fossi S, et al. Prediction of "awakening" and outcome in prolonged acute coma from severe traumatic brain injury: evidence for validity of short latency SEPs. Clin Neurophysiol. 2005;116(1):229-235.

60. Carter BG, Butt W. Are somatosensory evoked potentials the best predictor of outcome after severe brain injury? A systematic review. Intensive Care Med. 2005;31(6):765-775.

61. Gaetz M, Bernstein DM. The current status of electrophysiologic procedures for the assessment of mild traumatic brain injury. J Head Trauma Rehabil. 2001;16(4):386-405.

62. Shin DY, Ehrenberg B, Whyte J, Bach J, DeLisa JA. Evoked potential assessment: utility in prognosis of chronic head injury. Arch Phys Med Rehabil. 1989;70(3):189 - 193.

63. Polich J. P300 clinical utility and control of variability. J Clin Neurophysiol. 1998;15(1):14 - 33. 
64. Lew H, Chmiel R, Jerger J, Pomerantz JR, Jerger S. Electrophysiologic indices of Stroop and Garner interference reveal linguistic influences on auditory and visual processing. J Am Acad Audiol. 1997;8(2):104-118.

65. Polich J, Herbst KL. P300 as a clinical assay: rationale, evaluation, and findings. Int J Psychophysiol. 2000;38(1):3 - 19.

66. Polich J, Kok A. Cognitive and biological determinants of P300: an integrative review. Biol Psychol. 1995;41(2):103-146.

67. Viggiano MP. Event-related potentials in brain-injured patients with neuropsychological disorders: a review. J Clin Exp Neuropsychol. 1996;18(5):631 - 647.

68. Lew HL, Dikmen S, Slimp J, et al. Use of somatosensory-evoked potentials and cognitive event-related potentials in predicting outcomes of patients with severe traumatic brain injury. Am J Phys Med Rehabil. 2003;82(1):53 - 61.

69. Doi R, Morita K, Shigemori M, Tokutomi T, Maeda H. Characteristics of cognitive function in patients after traumatic brain injury assessed by visual and auditory event-related potentials. Am J Phys Med Rehabil. 2007;86(8):641 - 649 .

70. De Beaumont L, Théoret $\mathrm{H}$, Mongeon $\mathrm{D}$, et al. Brain function decline in healthy retired athletes who sustained their last sports concussion in early adulthood. Brain. 2009;132(pt 3):695 - 708.

71. Thériault $M$, De Beaumont $L$, Gosselin $N$, Filipinni $M$, Lassonde $M$. Electrophysiological abnormalities in well functioning multiple concussed athletes. Brain Inj. 2009;23(11):899-906.

72. Gosselin N, Thériault $\mathrm{M}$, Leclerc S, Montplaisir J, Lassonde M. Neurophysiological anomalies in symptomatic and asymptomatic concussed athletes. Neurosurgery. 2006;58(6):1151-1161; discussion 1151-1161.

73. Lavoie ME, Dupuis F, Johnston KM, Leclerc S, Lassonde M. Visual p300 effects beyond symptoms in concussed college athletes. J Clin Exp Neuropsychol. 2004;26(1):55-73.

74. Kobayashi M, Pascual-Leone A. Transcranial magnetic stimulation in neurology. Lancet Neurol. 2003;2(3):145-156.

75. Wagner T, Valero-Cabre A, Pascual-Leone A. Noninvasive human brain stimulation. Annu Rev Biomed Eng. 2007;9:527-565.

76. Reis J, Swayne OB, Vandermeeren Y, et al. Contribution of transcranial magnetic stimulation to the understanding of cortical mechanisms involved in motor control. J Physiol. 2008;586(2):325-351. 
77. Rossini PM, Barker AT, Berardelli A, et al. Non-invasive electrical and magnetic stimulation of the brain, spinal cord and roots: basic principles and procedures for routine clinical application. Report of an IFCN committee. Electroencephalogr Clin Neurophysiol. 1994; 91(2):79 - 92.

78. Chistyakov AV, Soustiel JF, Hafner H, Trubnik M, Levy G, Feinsod M. Excitatory and inhibitory corticospinal responses to transcranial magnetic stimulation in patients with minor to moderate head injury. J Neurol Neurosurg Psychiatry. 2001;70(5):580-587.

79. Hallett M. Transcranial magnetic stimulation and the human brain. Nature. 2000;406(6792):147-150.

80. Ziemann U, Rothwell JC, Ridding MC. Interaction between intracortical inhibition and facilitation in human motor cortex. J Physiol. 1996;496(pt 3):873 - 881.

81. De Beaumont L, Lassonde M, Leclerc S, Théoret H. Long-term and cumulative effects of sports concussion on motor cortex inhibition. Neurosurgery. 2007;61(2):329-336; discussion 336-327.

82. Livingston SC, Saliba EN, Goodkin HP, Barth JT, Hertel JN, Ingersoll CD. A preliminary investigation of motor evoked potential abnormalities following sport-related concussion. Brain Inj. 2010; 24(6):904 - 913.

83. Nardone R, Bergmann J, Kunz A, et al. Cortical excitability changes in patients with sleep-wake disturbances after traumatic brain injury. J Neurotrauma. 2011;28(7):1165-1171.

84. Tremblay S, de Beaumont L, Lassonde M, Théoret $\mathrm{H}$. Evidence for the specificity of intracortical inhibitory dysfunction in asymptomatic concussed athletes. J Neurotrauma. 2011;28(4):493-502.

85. Takeuchi N, Ikoma K, Chuma T, Matsuo Y. Measurement of transcallosal inhibition in traumatic brain injury by transcranial magnetic stimulation. Brain Inj. 2006;20(9):991 - 996.

86. Di Lazzaro V, Oliviero A, Profice P, et al. Muscarinic receptor blockade has differential effects on the excitability of intracortical circuits in the human motor cortex. Exp Brain Res. 2000;135(4): 455 - 461.

87. Kujirai T, Caramia MD, Rothwell JC, et al. Corticocortical inhibition in human motor cortex. J Physiol. 1993;471:501 - 519.

88. Wassermann EM. Risk and safety of repetitive transcranial magnetic stimulation: report and suggested guidelines from the International Workshop on the Safety of Repetitive Transcranial Magnetic Stimulation, June 5-7, 1996. Electroencephalogr Clin Neurophysiol. 1998;108(1):1 - 16. 
89. Ives JR, Rotenberg A, Poma R, Thut G, Pascual-Leone A. Electroencephalographic recording during transcranial magnetic stimulation in humans and animals. Clin Neurophysiol. 2006;117 (8):1870-1875.

90. Thut G, Ives JR, Kampmann F, Pastor MA, Pascual-Leone A. A new device and protocol for combining TMS and online recordings of EEG and evoked potentials. J Neurosci Methods. 2005;141(2): 207 - 217.

91. Thut G, Northoff G, Ives JR, et al. Effects of single-pulse transcranial magnetic stimulation (TMS) on functional brain activity: a combined event-related TMS and evoked potential study. Clin Neurophysiol. 2003;114(11):20712080.

92. Virtanen J, Ruohonen J, Naatanen R, Ilmoniemi RJ. Instrumentation for the measurement of electric brain responses to transcranial magnetic stimulation. Med Biol Eng Comput. 1999;37(3):322 - 326.

93. Pape TL, Rosenow J, Lewis G, et al. Repetitive transcranial magnetic stimulation-associated neurobehavioral gains during coma recovery. Brain Stimul. 2009;2(1):22-35.

94. Rossi S, Hallett M, Rossini PM, Pascual-Leone A. Safety, ethical considerations, and application guidelines for the use of transcranial magnetic stimulation in clinical practice and research. Clin Neurophysiol. 2009;120(12):2008-2039.

95. Nitsche MA, Paulus W. Excitability changes induced in the human motor cortex by weak transcranial direct current stimulation. J Physiol. 2000;527(pt 3):633-639.

96. Nitsche MA, Paulus W. Sustained excitability elevations induced by transcranial DC motor cortex stimulation in humans. Neurology. 2001;57(10):1899 - 1901.

97. NitscheMA,CohenLG,WassermannEM,etal.Transcranialdirect current stimulation: State of the art 2008. Brain Stimul. 2008;1(3): $206-223$.

98. Smith RB, Tiberi A, Marshall J. The use of cranial electrotherapy stimulation in the treatment of closed-head-injured patients. Brain Inj. 1994;8(4):357361.

99. TufailY,MatyushovA,BaldwinN,etal.Transcranialpulsedultra sound stimulates intact brain circuits. Neuron. 2010;66(5):681-694.

100. Yoo SS, Bystritsky A, Lee JH, et al. Focused ultrasound modulates regionspecific brain activity. Neuroimage. 2011;56(3):1267-1275.

101. VespaP.Whatistheoptimalthresholdforcerebralperfusionpressure following 
traumatic brain injury? Neurosurg Focus. 2003;15(6):E4.

102. Jaffres P, Brun J, Declety P, et al. Transcranial Doppler to detect on admission patients at risk for neurological deterioration following mild and moderate brain trauma. Intensive Care Med. 2005;31(6): 785 - 790.

103. Alexandrov AV. Ultrasound enhancement of fibrinolysis. Stroke. 2009;40(3)(suppl):S107-S110.

104. Vykhodtseva N, McDannold N, Hynynen K. Progress and problems in the application of focused ultrasound for blood-brain barrier disruption. Ultrasonics. 2008;48(4):279 - 296.

105. Bachtold MR, Rinaldi PC, Jones JP, Reines F, Price LR. Focused ultrasound modifications of neural circuit activity in a mammalian brain. Ultrasound Med Biol. 1998;24(4):557-565.

106. Karu T. Primary and secondary mechanisms of action of visible to near-IR radiation on cells. J Photochem Photobiol B. 1999;49(1):1-17.

107. Mochizuki-Oda N, Kataoka Y, Cui Y, Yamada H, Heya M, Awazu K. Effects of near-infra-red laser irradiation on adenosine triphosphate and adenosine diphosphate contents of rat brain tissue. Neurosci Lett. 2002;323(3):207210. 108.

108. Oron A, Oron U, Chen J, et al. Low-level laser therapy applied transcranially to rats after induction of stroke significantly reduces long-term neurological deficits. Stroke. 2006;37(10):2620-2624. 109.

109. Hashmi JT, Huang YY, Osmani BZ, Sharma SK, Naeser MA, Hamblin MR. Role of low-level laser therapy in neurorehabilitation. PM R. 2010;2(12)(suppl 2):S292-S305. 110.

110. Lampl Y, Zivin JA, Fisher M, et al. Infrared laser therapy for ischemic stroke: a new treatment strategy: results of the Neuro-Thera Effectiveness and Safety Trial-1 (NEST-1). Stroke. 2007;38(6): 1843 - 1849.

111. Zivin JA, Albers GW, Bornstein N, et al. Effectiveness and safety of transcranial laser therapy for acute ischemic stroke. Stroke. 2009; 40(4):1359 - 1364. 


\begin{tabular}{|c|c|c|c|c|}
\hline Rhythms & Frequency & $\begin{array}{l}\text { Main } \\
\text { distribution }\end{array}$ & $\begin{array}{l}\text { Recorded in } \\
\text { healthy }\end{array}$ & $\begin{array}{l}\text { Standard EEG } \\
\text { findings in TBI } \\
\text { patients }\end{array}$ \\
\hline Delta & $<4 \mathrm{~Hz}$ & & $\begin{array}{l}\text { Awake state, in the } \\
\text { very young and in } \\
\text { the elderly. Across } \\
\text { all ages during } \\
\text { slow-wave sleep }\end{array}$ & $\begin{array}{l}\text { Increased slow } \\
\text { waves in the delta } \\
\text { frequency band in } \\
\text { severe TBI }\end{array}$ \\
\hline Theta & $4-8 \mathrm{~Hz}$ & $\begin{array}{l}\text { Fronto- } \\
\text { central }\end{array}$ & $\begin{array}{l}\text { Resting or while } \\
\text { performing } \\
\text { moderately difficult } \\
\text { mental tasks; } \\
\text { enhanced by } \\
\text { drowsiness }\end{array}$ & $\begin{array}{l}\text { Rise in slow focal } \\
\text { or diffuse theta } \\
\text { activity }\end{array}$ \\
\hline Alpha & $8-13 \mathrm{~Hz}$ & Posterior & $\begin{array}{l}\text { Attenuated with } \\
\text { eye opening }\end{array}$ & $\begin{array}{l}\text { Immediate } \\
\text { decrease in the } \\
\text { mean frequency of } \\
\text { alpha waves }\end{array}$ \\
\hline $\mathrm{Mu}$ & $8-13 \mathrm{~Hz}$ & Central & $\begin{array}{l}\text { Attenuated with } \\
\text { contralateral } \\
\text { movement of an } \\
\text { extremity }\end{array}$ & \\
\hline Beta & $13-30 \mathrm{~Hz}$ & Frontal & $\begin{array}{l}\text { Mental, lingual, or } \\
\text { cognitive efforts }\end{array}$ & \\
\hline Gamma & $>30 \mathrm{~Hz}$ & $\begin{array}{l}\text { Diffuse, } \\
\text { central }\end{array}$ & $\begin{array}{l}\text { Higher cognitive } \\
\text { functions involving } \\
\text { perception, } \\
\text { attention, learning } \\
\text { and memory. } \\
\text { Assess the } \\
\text { temporal dynamics } \\
\text { of cortical } \\
\text { networks and their } \\
\text { interactions }\end{array}$ & \\
\hline
\end{tabular}

TABLE 1 Electroencephalography Rhythms and Their Significance in Healthy and TBI Populations 

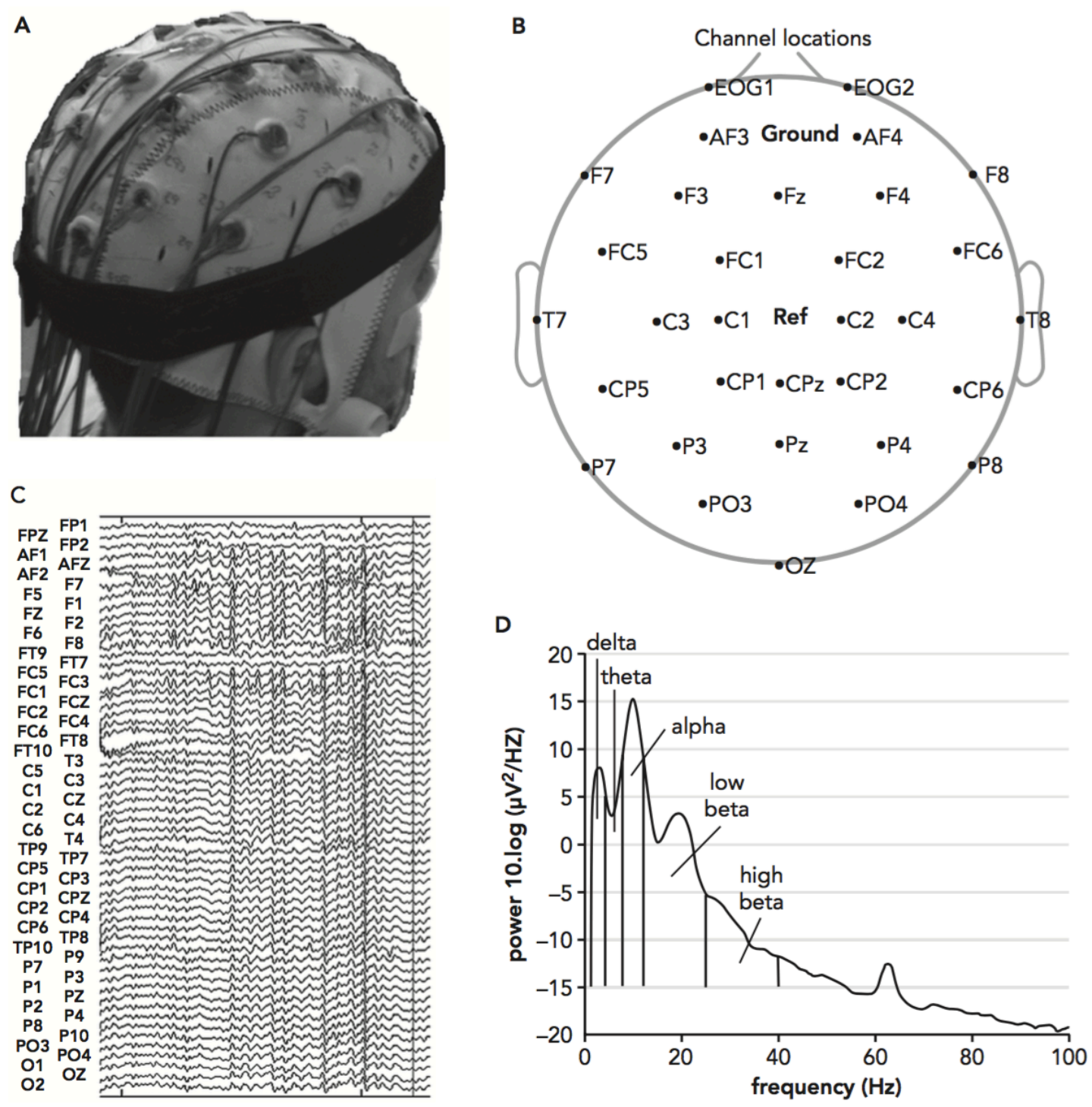

FIGURE 1 Electroencephalography (EEG). A, Setup of a 32 channels EEG system. B, Electrode positions on a topographical map. C, Raw recordings (conventional EEG). D, Example of the power spectrum in one channel (quantitative EEG). 


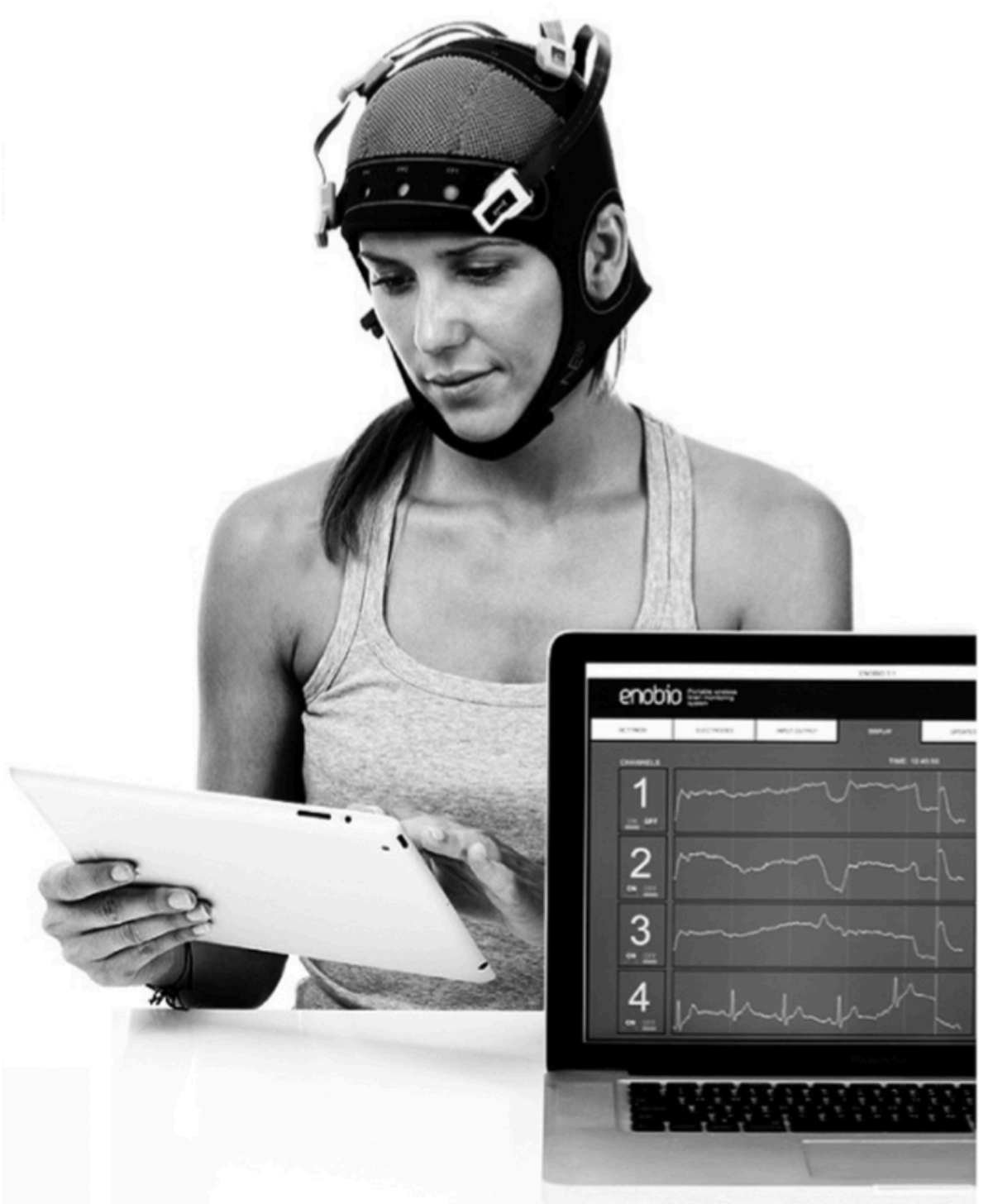

FIGURE 2 Setup of a wearable, modular, and wireless system of electroencephalography (EEG) recording (ENOBIO, Starlab, Barcelona, Spain). 


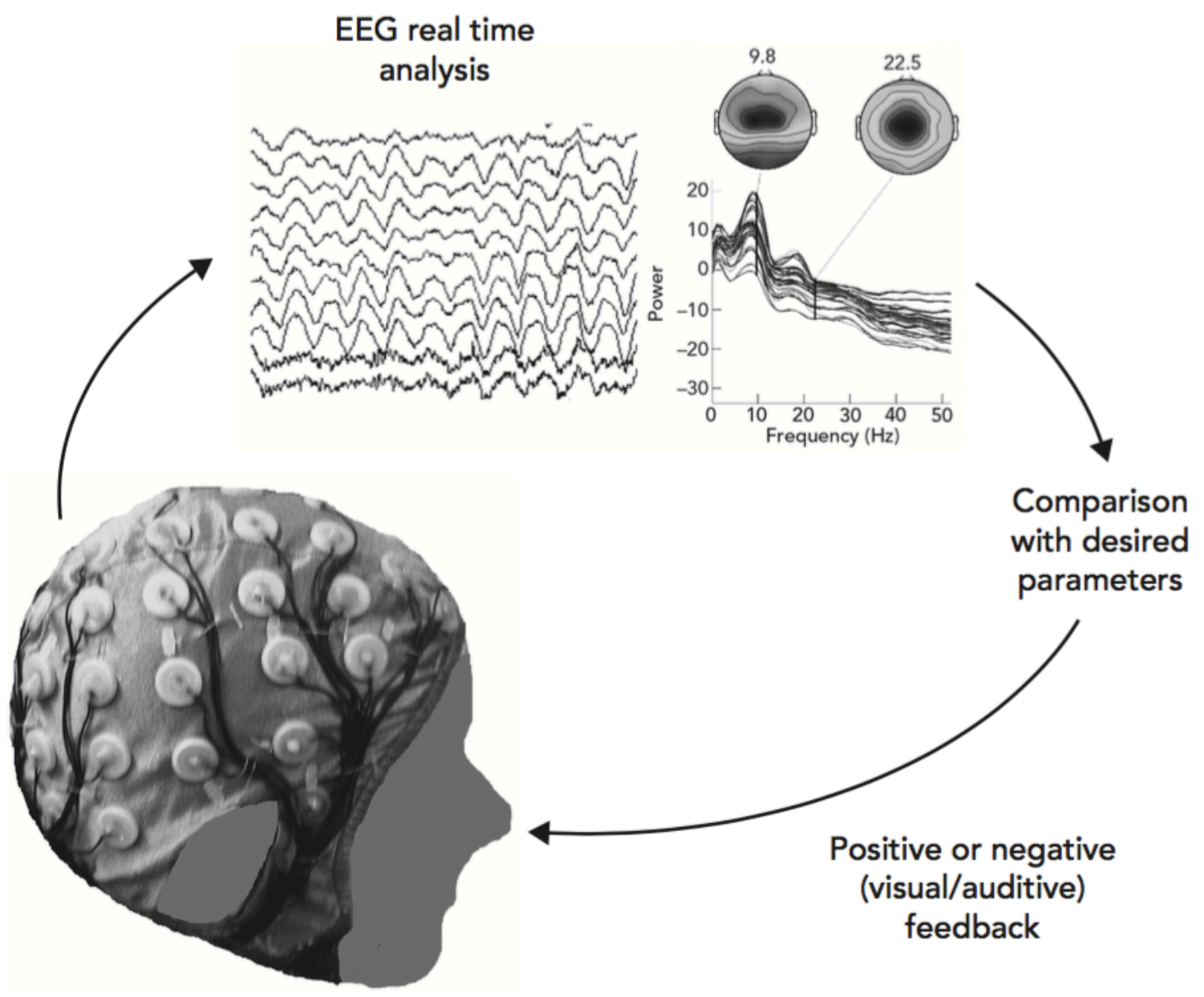

FIGURE 3 Schematic principle of electroencephalography (EEG) biofeedback. 


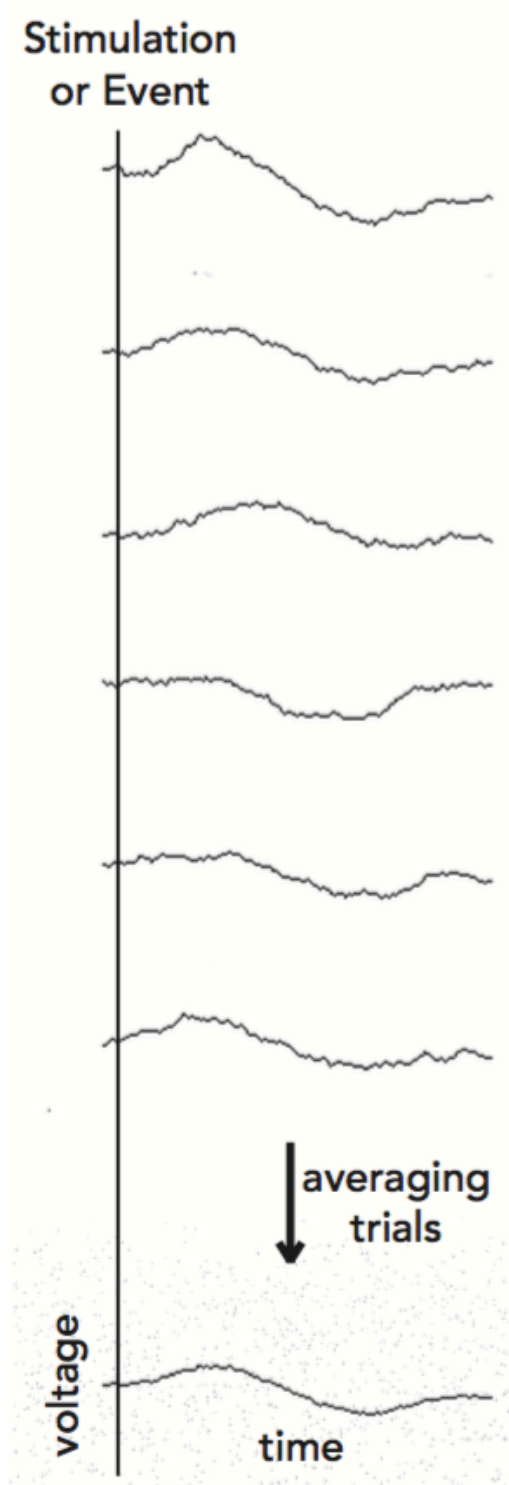

FIGURE 4 Principle of evoked potentials (EPs): stimulation or event-locked averaging of several trials allows the isolation of an electroencephalography (EEG) response to stimulation (EP) or event-related potential (ERP) from background EEG activity. 
Somatosensory Evoked Potentials after stimulation of Medial Nerve

Normal SEP

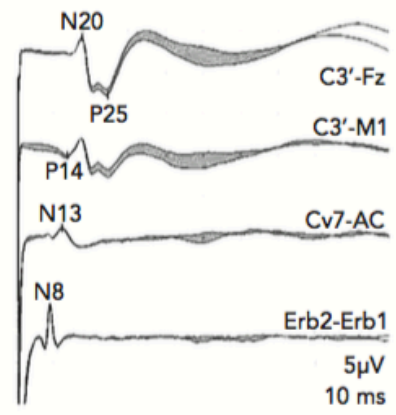

Pathological SEP

from an increased central condition time
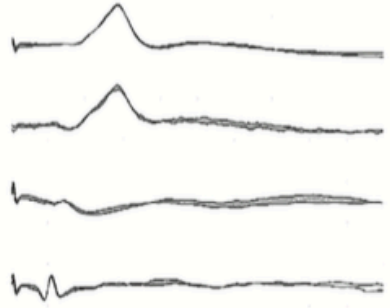

Absent cortical SEP

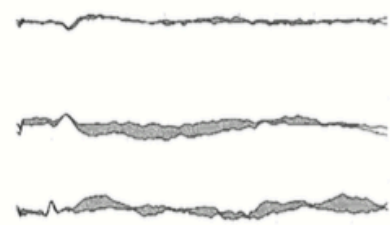

Event Related Potentials

in a Oddball Paradigm

Frequent Tone

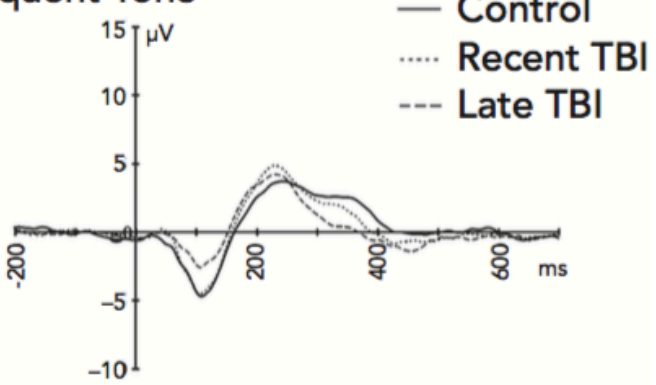

Rare Target Tone

(eliciting the P3b)

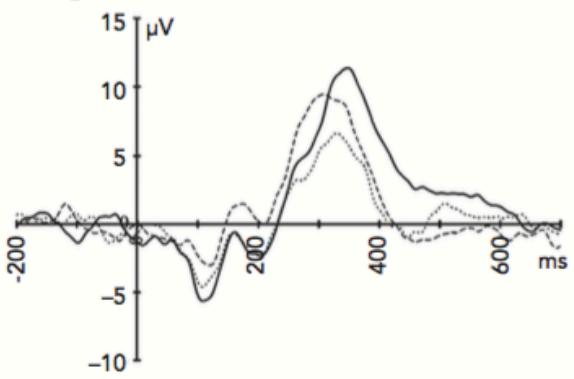

Rare Deviant Tone

(eliciting the P3a)

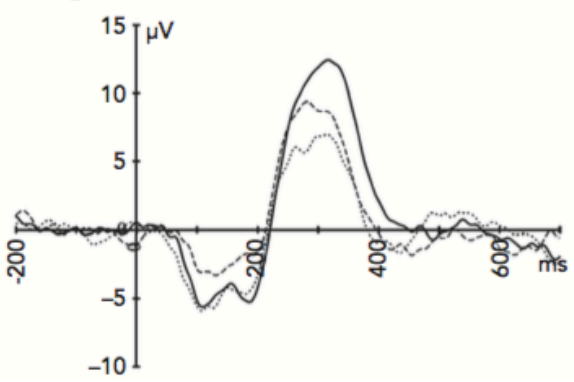

FIGURE 5 Illustration of somatosensory evoked potentials (SEPs) and event-related potentials (ERPs) in a traumatic brain injury (TBI) population compared to healthy controls. Left: SEPs. Adapted from Amantini et al. (57) with permission. Right: ERPs. Adapted from Thériault et al. (71) with permission. 
A Single pulse

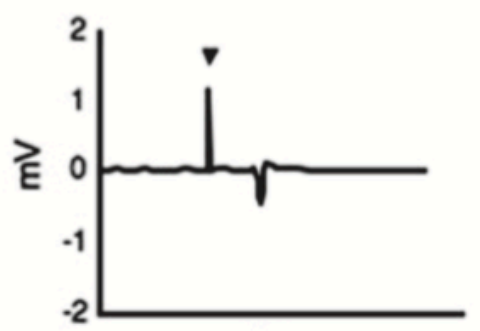

Time

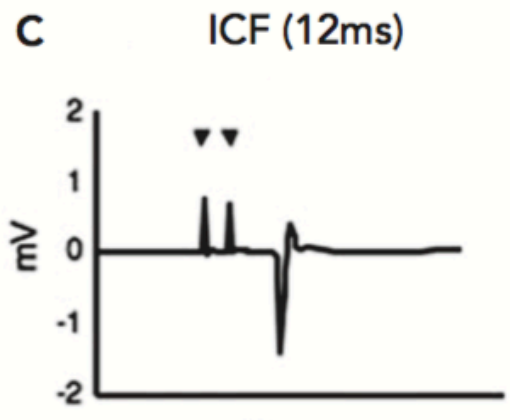

Time
B $\quad \mathrm{SICl}(2 \mathrm{~ms})$

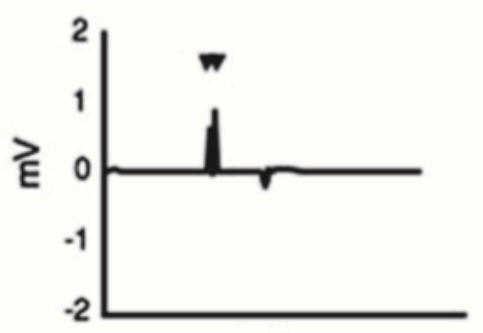

Time

D Ipsilateral silent period

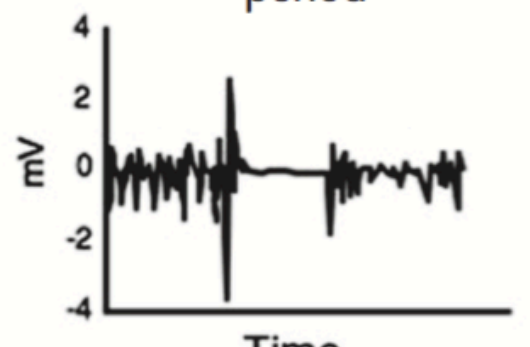

Time

FIGURE 6 Examples of transcranial magnetic stimulation (TMS) paradigms. A, A single pulse of TMS over M1 evokes an MEP recorded over the contralateral first dorsal interosseous. B, Two pulses separated by 2 ms evoke an MEP of smaller amplitude, revealing short interval intracortical inhibition (SICI). C, Two pulses separated by $12 \mathrm{~ms}$ evoke an MEP of larger amplitude, revealing intracortical facilitation (ICF). D, A pulse applied during a voluntary contraction of the contralateral hand evokes a pause in ongoing EMG activity following the MEP. Adapted from de Beaumont et al. (81) with permission.
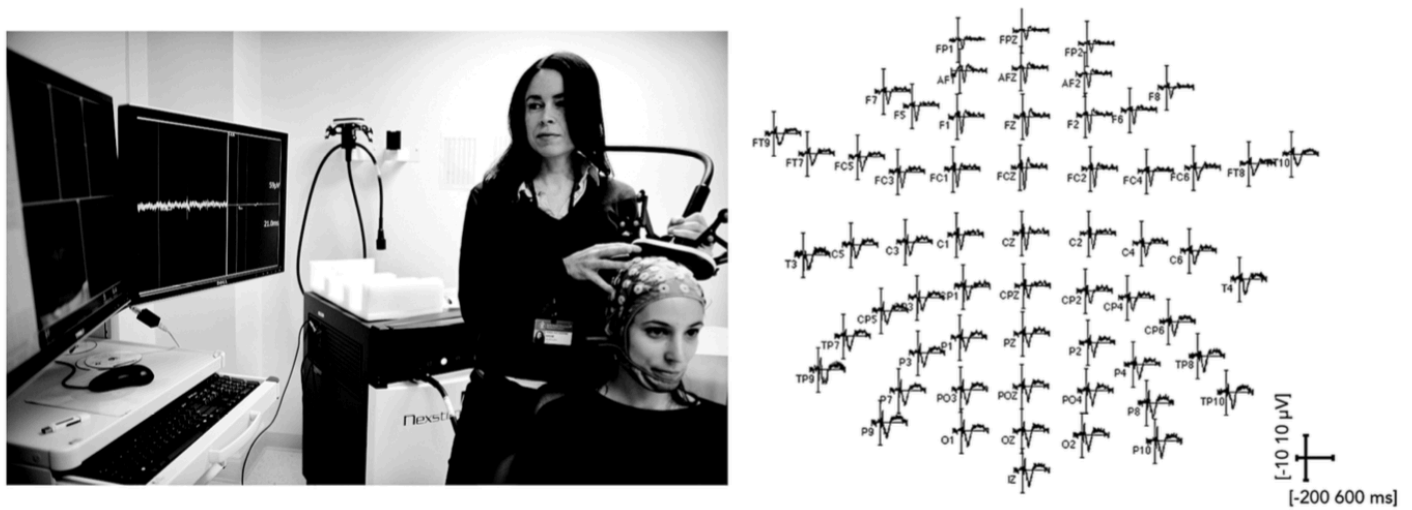

FIGURE 7 Transcranial magnetic stimulation (TMS) combined with electoencephalography (EEG). Left: a TMS-EEG system (Nexstim Oy, Helsinki, Finland). Right: TMS-evoked potentials for individual EEG channels. 